Mathematical Models and Methods in Applied Sciences Vol. 14, No. 4 (2004) 1-22

(c) World Scientific Publishing Company

\title{
MATHEMATICAL ANALYSIS OF NONLINEAR BONDED JOINT MODELS
}

\author{
FRANCOISE KRASUCKI \\ Laboratoire de Mécanique et de Génie Civil, \\ UMR 5508 Université Montpellier II, \\ Place Eugène Bataillon, 34695 Montpellier Cedex 5, France \\ krasucki@lmgc.univ-montp2.fr \\ ARNAUD MÜNCH \\ INRIA-Rocquencourt, Domaine de Voluceau, \\ B.P. 105, 78153 Le Chesnay Cedex, France \\ arnaud.munch@inria.fr \\ YVES OUSSET \\ Office National d'Etude et de Recherches Aérospatiales, \\ B.P. 72, 92322 Chatillon Cedex, France \\ yves.ousset@onera.fr \\ Received 23 October 2002 \\ Revised 23 September 2003 \\ Communicated by P. Podio Guidugli
}

\begin{abstract}
Within the framework of nonlinear elasticity, we consider the problem of two adherents joined along their common surface by a thin soft adhesive. Two stored energy functions are considered: the stored energy function of Saint Venant-Kirchhoff and the stored energy function of Ciarlet-Geymonat. Using the asymptotic expansion method, the limit energy associated to each of these stored energy functions is obtained. The aim of this paper is to give a rigorous mathematical analysis of the formally derived limit problem. We show that the limit problem associated to the Saint Venant-Kirchhoff case admits at least one solution and the limit problem associated to the Ciarlet-Geymonat case admits exactly one solution. An analytical comparison in the one-dimensional case and a three-dimensional numerical application are also presented.
\end{abstract}

Keywords: Bonded joint; nonlinear elasticity; asymptotic development.

\section{Introduction}

A pioneering paper presented at the Annual Meeting of the American Society of Mechanical Engineers by Goland and Reissner in 1943, deals with the determination of the stresses in cemented lap joints. This problem is formulated as one in plane strain and the authors obtain explicit solutions for two limiting cases. "(a) The case where the cement layer is so thin that its effects on the flexibility of the joint may 
be neglected; (b) the case in which the joint flexibility is mainly due to the cement layer. Case (b) is significant for the analysis of cemented metal sheets." (Ref. 1, p. A-17). In this case "the role played by the cemented layer is analogous to that of a system of infinitesimal coil springs positioned between the two plates." (Ref. 1, p. A-24). Since that time the modelling of bounded structure has progressed at least in two directions:

(1) In order to predict the delamination and the propagation of interlaminar cracks, more sophisticated models (nonlinear elasticity, plasticity, damage,...) have been introduced for the behavior of the adherents and of the adhesive.

(2) Formal asymptotic developments and mathematically assessed convergence theorems have been used in order to obtain a rational justification of the assumption on linear displacements and constant stresses in the adhesive.

The present paper aims to contribute mainly to the second direction under the following fundamental mechanical assumption:

(A) "The thickness of the adhesive, the flexibility of the adhesive and the external loads are small of the same order (under a natural adimensionalization)."

When all the materials are linearly elastic the assumption (A) corresponds to the case (B) of Goland and Reissner. For general linearly elastic materials, the rigorous convergence theorems of Refs. 2 and 3, prove that the most interesting results are obtained under the assumption (A).

In Sec. 2, we give a precise formulation of assumption (A), in terms of the small adimensional parameter $\varepsilon$, as far as it is concerned the thinness and the flexibility of the adhesive. For this, we at first introduce the domain obtained inserting the adhesive of thickness $\varepsilon h$ between the two adherents and then, we make explicit (see (2.8)) the assumption on the flexibility of the adhesive for two general nonlinear hyperelastic models: the Saint Venant-Kirchhoff model and a Ciarlet-Geymonat model. Both coincide with the usual Hooke law for small displacements (see e.g. Refs. 4 and 5) and then the assumption 2.8 is the most interesting (as has been proved e.g. in Refs. 2 and 3).

In Sec. 3, in order to complete the formal statement of assumption (A), we define at first an equivalent problem in a fixed domain and then introduce the assumption (3.2) on the external loads. Under the previous assumptions we look for a formal development of the critical points of the mechanical energy $I(\varepsilon, \mathbf{E}(\mathbf{v})$ and we identify the first term as a critical point of a suitable energy $I_{\mathrm{SV}}^{0}$, given by (3.7), for the Saint Venant-Kirchhoff model and $I_{\mathrm{CG}}^{0}$, given by (3.10), for Ciarlet-Geymonat model.

The next sections of the paper are devoted to a mathematical analysis of the two models. In Sec. 4, we study the Saint Venant-Kirchhoff model. The energy $I_{\mathrm{SV}}^{0}$ is continuous and differentiable for the strong topology of $V$ (defined in (4.3)). However, the energy is non-convex. In order to prove the existence of at least one critical point, we introduce the energy $\hat{I}_{\mathrm{SV}}^{0}$ defined on $\hat{V}$ (defined by (4.12)), where the adhesive has been replaced by the surface $S$ and the corresponding part 
of the energy by a surface energy. To a critical point of $\hat{I}_{\mathrm{SV}}^{0}$ one can associate a regular critical point of $I_{\mathrm{SV}}^{0}$, i.e. a critical point linear in the thickness variable of the adhesive. In order to prove that $\hat{I}_{\mathrm{SV}}^{0}$ has at least one critical point, we remark that the compact imbedding of $H^{1 / 2}(S)$ into $L^{2}(S)$ implies the lower semicontinuity for the weak topology of $\hat{V}$. We also prove that, for a suitable choice of the loads, $I_{\mathrm{SV}}^{0}$ may have many (non-regular, i.e. only piecewise linear in the thickness variable of the adhesive) critical points. We conjecture that only a regular critical point of $I_{\mathrm{SV}}^{0}$ corresponding to a critical point of $\hat{I}_{\mathrm{SV}}^{0}$ may be a minimum of $I_{\mathrm{SV}}^{0}$. The situation is completely different for the Ciarlet-Geymonat model $I_{\mathrm{CG}}^{0}$. Indeed, such a functional is convex and positive on a convex subset $U$ of $W$ (see (5.1) and (5.2)). Following the approach of J. Ball (Ref. 7), we can prove that there exists a unique minimum of $I_{\mathrm{CG}}^{0}$. As in Sec. 4 we also introduce a functional $\hat{I}_{\mathrm{CG}}^{0}$ defined on $\hat{U}$ (see (5.14) and deduce that $\mathbf{u}$ is linear in the thickness variable of the adhesive and it is associated to the unique minimum $\hat{\mathbf{u}}$ of $\hat{I}_{\mathrm{CG}}^{0}$.

The last two sections are devoted to the comparison of the models. In Sec. 6 such a comparison is done in a one-dimensional situation where the structure is composed of three bars. When the solution is linear in the adhesive one can explicitly compute the energy in the adhesive as a function of the strain. At last in Sec. 7 we test the asymptotic models for the single lap joint structure, one of the most studied in the literature. As in the one-dimensional case, the Ciarlet-Geymonat model is slightly softer than the Saint Venant-Kirchhoff model. For this last case a comparison is also done with the complete three-dimensional model. The numerically obtained rate of convergence is in good agreement with the assumption on the asymptotic expansion of $\mathbf{u}(\varepsilon)$.

\section{Problem Statement}

In the three-dimensional Euclidean space $\mathbb{E}^{3}$ referred to the orthonormal frame $\left(0 ; \mathbf{e}_{1}, \mathbf{e}_{2}, \mathbf{e}_{3}\right)$, let $\Omega^{-}$and $\Omega^{+}$be two disjoint domains with piecewise- $C^{2}$ boundaries $\partial \Omega^{+}$and $\partial \Omega^{-}$. Let $S=\partial \Omega^{+} \cap \partial \Omega^{-}$be the common part of the external surfaces which is assumed to have a positive $2 \mathrm{D}$ measure and which is assumed to be projectable onto the plane $\left\{x_{3}=0\right\}$. To "insert the adhesive", let $\Omega^{+}$(resp. $\Omega^{-}$) be moved in the $\mathbf{e}_{3}$ (resp. $-\mathbf{e}_{3}$ ) direction of an amount equal to the half-thickness, $\varepsilon h / 2$ of the joint. $\varepsilon$ is a small dimensionless parameter and $h$ is a global characteristic length (for example the diameter of $\Omega$ ). Then, let $\Omega_{\varepsilon}^{ \pm}=\left\{\mathbf{x}^{\varepsilon}:=\mathbf{x} \pm \varepsilon h / 2 \mathbf{e}_{3}, \mathbf{x} \in \Omega^{ \pm}\right\}$, $\Omega_{\varepsilon}^{m}=\left\{\mathbf{x}^{\varepsilon}:=\mathbf{x}+\varepsilon z / 2 \mathbf{e}_{3},-h<z<h, \mathbf{x} \in S\right\}, S_{\varepsilon}^{ \pm}=\left\{\mathbf{x}^{\varepsilon}:=\mathbf{x} \pm \varepsilon h / 2 \mathbf{e}_{3}, \mathbf{x} \in S\right\}$ and $\Omega_{\varepsilon}=\Omega_{\varepsilon}^{+} \cup \Omega_{\varepsilon}^{-} \cup \Omega_{\varepsilon}^{m} \cup S_{\varepsilon}^{+} \cup S_{\varepsilon}^{-}$be the physical reference configuration of the assembly (see Fig. 1(a)). Following the usual terminology, $\Omega_{\varepsilon}^{+}$and $\Omega_{\varepsilon}^{-}$are filled by the adherents, while $\Omega_{\varepsilon}^{m}$ is filled by the adhesive. The structure is fixed on the parts $\Gamma_{u}^{+} \subset \partial \Omega_{\varepsilon}^{+}$, resp. $\Gamma_{u}^{-} \subset \partial \Omega_{\varepsilon}^{-}$. The external boundary of the adhesive is traction-free. The complementary part of the boundary $\Gamma_{f}$ is submitted to surface loads $\mathbf{f}_{\varepsilon}^{d}$. Obviously, one can consider other types of boundary conditions 


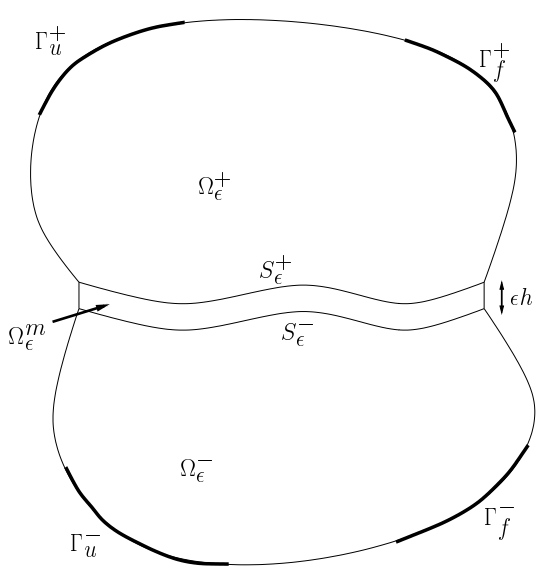

(a)

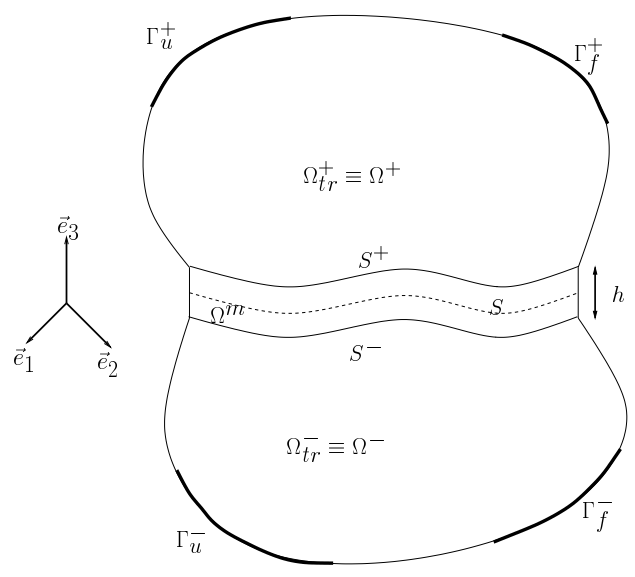

(b)

Fig. 1. Bonded assembly: (a) the physical problem $\left(\Omega_{\varepsilon}\right)$, (b) the rescaled problem $\left(\Omega_{\mathrm{tr}} \equiv \Omega\right)$.

(e.g. a complementary combination of components of the stress vector and of the displacement as in the numerical example of Sec. 7).

The study is within the framework of nonlinear elasticity and we consider that each body is made of an isotropic hyperelastic material. A Lagrangian description is used to describe the deformation of the structure: we design by $\mathbf{E}$ the Green-Lagrange strain tensor defined by

$$
\mathbf{E}(\mathbf{u})=\frac{1}{2}\left(\mathbf{F}^{t}(\mathbf{u}) \mathbf{F}(\mathbf{u})-\mathbf{I}_{3}\right) ; \quad \mathbf{F}(\mathbf{u})=\nabla \mathbf{u}+\mathbf{I}_{3},
$$

where $\mathbf{F}$ is the gradient of the mapping $\mathbf{x}^{\varepsilon} \rightarrow \mathbf{x}^{\varepsilon}+\mathbf{u}^{\varepsilon}\left(\mathbf{x}^{\varepsilon}\right), \mathbf{u}^{\varepsilon}$ the displacement field, $\mathbf{I}_{3}$ is the identity tensor of $\mathbb{E}^{3}$ and $\nabla \mathbf{u}^{\varepsilon}=\left(\frac{\partial u^{\varepsilon}}{\partial x^{\varepsilon}}\right)$. Finally, we denote by $\boldsymbol{\sigma}^{\varepsilon}\left(\mathbf{x}^{\varepsilon}\right)$ the second Piola-Kirchhoff stress, a symmetric tensor-valued field. The hyperelastic behavior is given by the relation

$$
\boldsymbol{\sigma}^{\varepsilon}\left(\mathbf{x}^{\varepsilon}\right)=\frac{\partial \breve{W}^{\varepsilon}}{\partial \mathbf{E}}\left(\mathbf{x}^{\varepsilon}, \mathbf{E}\left(\mathbf{u}^{\varepsilon}\left(\mathbf{x}^{\varepsilon}\right)\right)\right)
$$

where $\breve{W}^{\varepsilon}$ designs the stored energy function. In this work, we are concerned with two stored energy functions. These are

- the stored energy function of Saint Venant-Kirchhoff (Ref. 4):

$$
\begin{aligned}
\hat{W}_{\mathrm{SV}}^{\varepsilon}(\mathbf{F})= & \breve{W}_{\mathrm{SV}}^{\varepsilon}(\mathbf{E})=\frac{\lambda^{\varepsilon}}{2}(\operatorname{Tr} \mathbf{E})^{2}+\mu^{\varepsilon} \operatorname{Tr} \mathbf{E}^{2} \\
= & -\frac{3 \lambda^{\varepsilon}+2 \mu^{\varepsilon}}{4}\|\mathbf{F}\|^{2}+\frac{\lambda^{\varepsilon}+2 \mu^{\varepsilon}}{8} \operatorname{Tr}\left(\left(\mathbf{F}^{t} \mathbf{F}\right)^{2}\right) \\
& +\frac{\lambda^{\varepsilon}}{4}\|\operatorname{cof} \mathbf{F}\|^{2}+\frac{6 \mu^{\varepsilon}+9 \lambda^{\varepsilon}}{8},
\end{aligned}
$$


$\left(\|\mathbf{F}\|^{2}=\operatorname{Tr}\left(\mathbf{F}^{t} \mathbf{F}\right),\|\operatorname{cof} \mathbf{F}\|^{2}=\frac{1}{2}\left(\|\mathbf{F}\|^{4}-\operatorname{Tr}\left(\mathbf{F}^{t} \mathbf{F}\right)^{2}\right)\right.$ where $\operatorname{Tr}$ designs the trace operator), which is the simplest hyperelastic stored energy function valid for small strains;

- the stored energy function of Ciarlet-Geymonat (Ref. 5):

$$
\hat{W}_{\mathrm{CG}}^{\varepsilon}(\mathbf{F})=a\|\mathbf{F}\|^{2}+b\|\operatorname{cof} \mathbf{F}\|^{2}+\Gamma(\operatorname{det} \mathbf{F})+e,
$$

with $\Gamma(\delta)=c \delta^{2}-d \log (\delta)(\delta>0)$ and with the coefficients $a, b, c, d \in \mathbb{R}_{+}^{*}$ and $e \in \mathbb{R}$ satisfying the relations

$$
\begin{cases}3 a+3 b+\Gamma(1)+e=0, & 2 a+4 b+\Gamma^{\prime}(1)=0, \\ 2 b+\frac{1}{2} \Gamma^{\prime}(1)+\frac{1}{2} \Gamma^{\prime \prime}(1)=\frac{\lambda^{\varepsilon}}{2}, & -2 b-\Gamma^{\prime}(1)=\mu^{\varepsilon},\end{cases}
$$

leading to:

$$
\begin{aligned}
\breve{W}_{\mathrm{CG}}^{\varepsilon}(\mathbf{E})= & \left(\frac{\lambda^{\varepsilon}}{2}+\mu^{\varepsilon}\right) \operatorname{Tr} \mathbf{E}+\frac{\lambda^{\varepsilon}}{2}\left[(\operatorname{Tr} \mathbf{E})^{2}-\operatorname{Tr} \mathbf{E}^{2}\right] \\
& +\lambda^{\varepsilon} \operatorname{det} \mathbf{E}-\frac{\lambda^{\varepsilon}+2 \mu^{\varepsilon}}{4} \log (\operatorname{det}(I+2 \mathbf{E})) .
\end{aligned}
$$

Remark 1. For small strains, the stored energy function of Ciarlet-Geymonat $\breve{W}_{\text {CG }}^{\varepsilon}$ coincides, for a suitable choice of the coefficients as in (2.5), with the stored energy function $\breve{W}_{\mathrm{SV}}^{\varepsilon}$ (see Ref. 5):

$$
\breve{W}_{\mathrm{CG}}^{\varepsilon}(\mathbf{E})=\breve{W}_{\mathrm{SV}}^{\varepsilon}(\mathbf{E})+o(\|\mathbf{E}\|) .
$$

Remark 2. The stored energy function of Ciarlet-Geymonat is defined for det $\mathbf{F}>0$, which corresponds to the local orientation-preserving condition.

As in Ref. 6, the adhesive is assumed to be soft, i.e. its elastic stiffness is small with respect to the ones of the adherents. This is stressed by introducing $\lambda^{m}, \lambda^{+}, \lambda^{-}$ (resp. $\mu^{m}, \mu^{+}, \mu^{-}$) independent of $\varepsilon$ and of same order, such that:

$$
\left\{\begin{array}{lll}
\lambda^{\varepsilon}=\lambda^{ \pm}, & \mu^{\varepsilon}=\mu^{ \pm} & \text {in } \Omega_{\varepsilon}^{ \pm} \\
\lambda^{\varepsilon}=\varepsilon \lambda^{m}, & \mu^{\varepsilon}=\varepsilon \mu^{m} & \text { in } \Omega_{\varepsilon}^{m}
\end{array}\right.
$$

Finally, the mechanical energy is defined as

$$
I^{\varepsilon}(\mathbf{E}(\mathbf{v}))=\int_{\Omega^{\varepsilon}} \breve{W}^{\varepsilon}\left(\mathbf{x}^{\varepsilon}, \mathbf{E}(\mathbf{v})\right) d \mathbf{x}^{\varepsilon}-L^{\varepsilon}(\mathbf{v}),
$$

$L^{\varepsilon}$ being a linear form associated to the surface load:

$$
L^{\varepsilon}(\mathbf{v})=\int_{\Gamma_{f}} \mathbf{f}_{\varepsilon}^{d} \cdot \mathbf{v} d \Gamma
$$

In the case of $\breve{W}_{\mathrm{SV}}^{\varepsilon}$, the corresponding energy, seen as a function of the displacement field $\mathbf{u}^{\varepsilon}$, is defined on the functional space

$$
V_{\mathrm{SV}}\left(\Omega^{\varepsilon}\right)=\left\{\mathbf{v} \in\left(W^{1,4}\left(\Omega^{\varepsilon}\right)\right)^{3}, \mathbf{v}=0 \text { on } \Gamma_{u}^{ \pm}\right\},
$$


whereas in the case of $\breve{W}_{\mathrm{CG}}$, the corresponding space is:

$$
V_{\mathrm{CG}}\left(\Omega^{\varepsilon}\right)=\left\{\mathbf{v} \in\left(W^{1,6}\left(\Omega^{\varepsilon}\right)\right)^{3}, \operatorname{det} \mathbf{F}(\mathbf{v})>0, \mathbf{v}=0 \text { on } \Gamma_{u}^{ \pm}\right\} .
$$

Remark 3. The parameter $\varepsilon$ being fixed, the solution $\mathbf{u}^{\varepsilon}$ is, at least formally, a critical point of the mechanical energy. In the case of the stored energy function of Ciarlet-Geymonat, thanks to J. Ball's results, we know that there exists a minimum (Ref. 9). This point is still an open question for the energy associated to $\breve{W}_{\text {SV }}^{\varepsilon}$ (Ref. 2).

The aim of this paper is to give a rigorous mathematical analysis of the limit problem obtained formally in Refs. 6 and 8. In the text, the repeated summation convention is used: Latin $i, j, k, \ldots$ (resp. Greek $\alpha, \beta, \ldots$ ) indices take their values in the set $\{1,2,3\}$ (resp. $\{1,2\})$.

\section{Rescaling and Asymptotic Expansion}

(i) In order to make apparent the dependence on $\varepsilon$ of the problem, we define, following the approach of Ciarlet and Destuynder (Ref. 9), an equivalent problem in the fixed domain $\Omega_{\text {tr }}$ (see Fig. 1(b)). For this purpose, we set $\pi^{\varepsilon}: \mathbf{x}=\left(x_{1}, x_{2}, x_{3}\right) \in$ $\Omega_{\mathrm{tr}} \rightarrow \mathbf{x}^{\varepsilon}=\left(x_{1}^{\varepsilon}, x_{2}^{\varepsilon}, x_{3}^{\varepsilon}\right) \in \Omega_{\varepsilon}$ defined by:

$$
\begin{cases}\pi^{\varepsilon}\left(x_{1}, x_{2}, x_{3}\right)=\left(x_{1}, x_{2}, x_{3}-\frac{h}{2}(1-\varepsilon)\right) \in \Omega_{\varepsilon}^{+}, & \text {for } \mathbf{x} \in \Omega_{\mathrm{tr}}^{+}, \\ \pi^{\varepsilon}\left(x_{1}, x_{2}, x_{3}\right)=\left(x_{1}, x_{2}, \varepsilon x_{3}\right) \in \Omega_{\varepsilon}^{m}, & \text { for } \mathbf{x} \in \Omega^{m}, \\ \pi^{\varepsilon}\left(x_{1}, x_{2}, x_{3}\right)=\left(x_{1}, x_{2}, x_{3}+\frac{h}{2}(1-\varepsilon)\right) \in \Omega_{\varepsilon}^{-}, & \text {for } \mathbf{x} \in \Omega_{\mathrm{tr}}^{-},\end{cases}
$$

with $\Omega_{\mathrm{tr}}^{ \pm}=\left\{\mathbf{x} \pm \frac{h}{2} \mathbf{e}_{3}, \mathbf{x} \in \Omega^{ \pm}\right\}, \Omega^{m}=\left\{\mathbf{x}+\frac{z}{2} \mathbf{e}_{3},-h<z<h, \mathbf{x} \in S\right\}, S^{ \pm}=$ $\left\{\mathbf{x} \pm \frac{h}{2} \mathbf{e}_{3}, \mathbf{x} \in S\right\}$. In order to simplify the notations, we identify $\Omega_{\mathrm{tr}}^{+}$with $\Omega^{+}$ and $\Omega_{\mathrm{tr}}^{-}$with $\Omega^{-}$. At last, we set $\bar{\Omega}=\bar{\Omega}^{+} \cup \bar{\Omega}^{-} \cup \bar{\Omega}^{m}$. The displacement field and the elastic properties of the bodies are then defined without rescaling: $\mathbf{u}(\varepsilon, \mathbf{x})=$ $\mathbf{u}^{\varepsilon}\left(\mathbf{x}^{\varepsilon}\right)=\mathbf{u}^{\varepsilon} \circ \pi^{\varepsilon}(\mathbf{x}), \lambda(\varepsilon, \mathbf{x})=\lambda^{\varepsilon}\left(\mathbf{x}^{\varepsilon}\right)$ and $\mu(\varepsilon, \mathbf{x})=\mu^{\varepsilon}\left(\mathbf{x}^{\varepsilon}\right)$ whereas the following rescaling of the load is performed:

$$
\mathbf{f}_{\varepsilon}^{d}\left(\mathbf{x}^{\varepsilon}\right)=\varepsilon \mathbf{f}^{d}(\mathbf{x}) .
$$

Let us also recall the usual relations $\frac{\partial}{\partial x_{\alpha}^{\varepsilon}}\left(\phi^{\varepsilon}\left(\mathbf{x}^{\varepsilon}\right)\right)=\frac{\partial}{\partial x_{\alpha}}(\phi(\mathbf{x})), \frac{\partial}{\partial x_{3}^{\varepsilon}}\left(\phi^{\varepsilon}\left(\mathbf{x}^{\varepsilon}\right)\right)=$ $\varepsilon^{-1} \frac{\partial}{\partial x_{3}}(\phi(\mathbf{x}))$ and $\int_{\Omega_{\varepsilon}^{m}} \phi^{\varepsilon}\left(\mathbf{x}^{\varepsilon}\right) d \mathbf{x}^{\varepsilon}=\varepsilon \int_{\Omega^{m}} \phi^{\varepsilon} \circ \pi^{\varepsilon}(\mathbf{x}) d \mathbf{x} \equiv \varepsilon \int_{\Omega^{m}} \phi(\mathbf{x}) d \mathbf{x}$ for any differentiable function $\phi^{\varepsilon}$ defined in $\Omega_{\varepsilon}^{m}$. Then the equivalent problem is to look for a critical point of the following energy defined in $\Omega$ :

$$
I(\varepsilon, \mathbf{E}(\mathbf{v}))=\int_{\Omega} \breve{W}(\varepsilon, \mathbf{x}, \mathbf{E}(\mathbf{v})) d \mathbf{x}-L(\varepsilon, \mathbf{v}),
$$

where $L(\varepsilon, \mathbf{v})=\varepsilon \int_{\Gamma_{f}} \mathbf{f}^{d} \cdot \mathbf{v} d \Gamma$. 
(ii) In the Saint Venant-Kirchhoff case, we obtain

$$
\begin{aligned}
\int_{\Omega} \breve{W}_{\mathrm{SV}}(\varepsilon, \mathbf{x}, \mathbf{E}(\mathbf{v})) d \mathbf{x}= & \int_{\Omega^{ \pm}}\left(\frac{\lambda^{ \pm}}{2}(\operatorname{Tr} \mathbf{E}(\mathbf{v}))^{2}+\mu^{ \pm} \operatorname{Tr}(\mathbf{E}(\mathbf{v}))^{2}\right) d \Omega \\
& +\int_{\Omega^{m}}\left(\varepsilon^{-2} W^{-2}(\mathbf{v})+\varepsilon^{-1} W^{-1}(\mathbf{v})+W^{0}(\mathbf{v})\right. \\
& \left.+\varepsilon^{1} W^{1}(\mathbf{v})+\varepsilon^{2} W^{2}(\mathbf{v})\right) d \Omega
\end{aligned}
$$

with

$$
\begin{aligned}
W^{-2}(\mathbf{v})= & \frac{1}{8}\left(\lambda^{m}+2 \mu^{m}\right)\left(v_{k, 3} v_{k, 3}\right)^{2}, \\
W^{-1}(\mathbf{v})= & \frac{1}{2}\left(\lambda^{m}+2 \mu^{m}\right) v_{k, 3} v_{k, 3} v_{3,3}, \\
W^{0}(\mathbf{v})= & \frac{1}{2}\left(\lambda^{m}+2 \mu^{m}\right) v_{3,3}^{2}+\frac{\lambda^{m}}{2} v_{k, 3} v_{k, 3}\left(v_{\alpha, \alpha}+\frac{1}{2} v_{p, \alpha} v_{p, \alpha}\right) \\
& +\frac{\mu^{m}}{2}\left(v_{\alpha, 3}+v_{k, \alpha} v_{k, 3}\right)^{2}, \\
W^{1}(\mathbf{v})= & \lambda^{m}\left(v_{\alpha, \alpha}+\frac{1}{2} v_{k, \alpha} v_{k, \alpha}\right) v_{3,3}+\mu^{m} v_{3, \alpha}\left(v_{\alpha, 3}+v_{k, \alpha} v_{k, 3}\right), \\
W^{2}(\mathbf{v})= & \frac{\lambda^{m}}{2}\left(v_{\alpha, \alpha}+\frac{1}{2} v_{k, \alpha} v_{k, \alpha}\right)^{2}+\frac{\mu^{m}}{2} v_{3, \alpha} v_{3, \alpha} \\
& +\frac{\mu^{m}}{4}\left(v_{\alpha, \beta}+v_{\beta, \alpha}+v_{k, \alpha} v_{k, \beta}\right)\left(v_{\alpha, \beta}+v_{\beta, \alpha}+v_{l, \alpha} v_{l, \beta}\right) .
\end{aligned}
$$

This suggests to look formally for a solution $\mathbf{u}(\varepsilon, \mathbf{x})$ such that:

$$
\mathbf{u}(\varepsilon, \mathbf{x})=\mathbf{u}^{0}(\mathbf{x})+\varepsilon \mathbf{u}^{1}(\mathbf{x})+\cdots .
$$

One can easily show that, thanks to $(3.2), \mathbf{u}^{0} \equiv 0$ in $\Omega$ leading to the expansion

$$
I_{\mathrm{SV}}(\varepsilon, \mathbf{E}(\mathbf{u}(\varepsilon)))=\varepsilon^{2} I_{\mathrm{SV}}^{0}\left(\mathbf{u}^{1}\right)+O\left(\varepsilon^{3}\right) .
$$

As a consequence, the initial problem is, for the leading term, formally reduced to the search of the critical points of the following energy:

$$
I_{\mathrm{SV}}^{0}(\mathbf{v})=\int_{\Omega} \breve{W}^{0}(\mathbf{x}, \mathbf{E}(\mathbf{v})) d \mathbf{x}-L^{0}(\mathbf{v})
$$

with

$$
\begin{aligned}
\int_{\Omega} \breve{W}^{0}(\mathbf{x}, \mathbf{E}(\mathbf{v})) d \mathbf{x} \\
=\int_{\Omega^{ \pm}}\left(\frac{\lambda^{ \pm}}{2}(\operatorname{Tr} \mathbf{e}(\mathbf{v}))^{2}+\mu^{ \pm} \operatorname{Tr}(\mathbf{e}(\mathbf{v}))^{2}\right) d \Omega \\
\quad+\int_{\Omega^{m}}\left(W^{-2}(\mathbf{v})+W^{-1}(\mathbf{v})+\frac{1}{2}\left(\lambda^{m}+2 \mu^{m}\right) v_{3,3}^{2}+\frac{\mu^{m}}{2} v_{\alpha, 3} v_{\alpha, 3}\right) d \Omega
\end{aligned}
$$


where $L^{0}(\mathbf{v})=\int_{\Gamma_{f}} \mathbf{f}^{d} \cdot \mathbf{v} d \Gamma$ and $\mathbf{e}(\mathbf{v})=\frac{1}{2}\left(\nabla \mathbf{v}+(\nabla \mathbf{v})^{t}\right)$ the linear part of the strain tensor $\mathbf{E}$.

(iii) In a similar way for the Ciarlet-Geymonat case, we assume $\mathbf{u}(\varepsilon, \mathbf{x})=$ $\varepsilon \mathbf{u}^{1}(\mathbf{x})+\cdots$ and we obtain

$$
I_{\mathrm{CG}}(\varepsilon, \mathbf{E}(\mathbf{u}(\varepsilon)))=\varepsilon^{2} I_{\mathrm{CG}}^{0}\left(\mathbf{u}^{1}\right)+O\left(\varepsilon^{3}\right),
$$

with

$$
\begin{aligned}
I_{\mathrm{CG}}^{0}(\mathbf{v})= & \int_{\Omega^{ \pm}}\left(\frac{\lambda^{ \pm}}{2}(\operatorname{Tr} \mathbf{e}(\mathbf{v}))^{2}+\mu^{ \pm} \operatorname{Tr}(\mathbf{e}(\mathbf{v}))^{2}\right) d \Omega \\
& +\int_{\Omega^{m}}\left(\mu^{m}+\frac{\lambda^{m}}{2}\right)\left(\frac{1}{2} v_{3,3}^{2}+v_{3,3}-\log \left(1+v_{3,3}\right)\right) d \Omega \\
& +\int_{\Omega^{m}} \frac{\mu^{m}}{2} v_{\alpha, 3} v_{\alpha, 3} d \Omega-\int_{\Gamma_{f}} \mathbf{f}^{d} \cdot \mathbf{v} d \Gamma .
\end{aligned}
$$

In the following, the quantity $\mathbf{u}^{1}$ will be simply noted as $\mathbf{u}$.

\section{Study of the Limit Problem: The Saint Venant-Kirchhoff Case}

(i) From the relation

$$
\begin{aligned}
& W^{-2}(\mathbf{v})+W^{-1}(\mathbf{v})+\frac{1}{2}\left(\lambda^{m}+2 \mu^{m}\right) v_{3,3}^{2}+\frac{\mu^{m}}{2} v_{\alpha, 3} v_{\alpha, 3} \\
& \quad=\frac{1}{8}\left(\lambda^{m}+2 \mu^{m}\right)\left(\left(v_{1,3}^{2}+v_{2,3}^{2}\right)+\left(v_{3,3}+1\right)^{2}-1\right)^{2}+\frac{\mu^{m}}{2}\left(v_{1,3}^{2}+v_{2,3}^{2}\right),
\end{aligned}
$$

the limit energy becomes simply:

$$
\begin{aligned}
I_{\mathrm{SV}}^{0}(\mathbf{v})= & \int_{\Omega^{ \pm}}\left(\frac{\lambda^{ \pm}}{2}(\operatorname{Tr} \mathbf{e}(\mathbf{v}))^{2}+\mu^{ \pm} \operatorname{Tr}(\mathbf{e}(\mathbf{v}))^{2}\right) d \Omega \\
& +\frac{1}{8} \int_{\Omega^{m}}\left(\lambda^{m}+2 \mu^{m}\right)\left(\left(v_{1,3}^{2}+v_{2,3}^{2}\right)+\left(v_{3,3}+1\right)^{2}-1\right)^{2} d \Omega \\
& +\frac{1}{2} \int_{\Omega^{m}} \mu^{m}\left(v_{1,3}^{2}+v_{2,3}^{2}\right) d \Omega-L^{0}(\mathbf{v}) .
\end{aligned}
$$

Then, assuming $\mathbf{f}^{d} \in\left(L^{2}\left(\Gamma_{f}\right)\right)^{3}$, the functional $I_{\mathrm{SV}}^{0}$ is defined and continuous on the space:

$$
\begin{aligned}
V=\left\{\mathbf{v}^{-} \in\left(H^{1}\left(\Omega^{-}\right)\right)^{3}, \mathbf{v}^{+} \in\left(H^{1}\left(\Omega^{+}\right)\right)^{3}, \mathbf{v}^{m} \in\left(L^{4}\left(\Omega^{m}\right)\right)^{3}, v_{k, 3}^{m} \in L^{4}\left(\Omega^{m}\right),\right. \\
\left.\mathbf{v}_{\mid S^{+}}^{+}=\mathbf{v}_{\mid S^{+}}^{m} ; \mathbf{v}_{\mid S^{-}}^{-}=\mathbf{v}_{\mid S^{-}}^{m}, \mathbf{v}^{ \pm}=0 \text { on } \Gamma_{u}^{ \pm}\right\},
\end{aligned}
$$

where $\mathbf{v}^{+}, \mathbf{v}^{-}$and $\mathbf{v}^{m}$ are the restrictions of $\mathbf{v}$ to $\Omega^{+}, \Omega^{+}$and $\Omega^{m}$, respectively. $V$ is endowed with the natural norm $\|\mathbf{v}\|_{V}$ such that:

$$
\|\mathbf{v}\|_{V}^{2}=\left\|\mathbf{v}^{+}\right\|_{\left(H^{1}\left(\Omega^{+}\right)\right)^{3}}^{2}+\left\|\mathbf{v}^{-}\right\|_{\left(H^{1}\left(\Omega^{-}\right)\right)^{3}}^{2}+\left\|\mathbf{v}^{m}\right\|_{\left(L^{4}\left(\Omega^{m}\right)\right)^{3}}^{2}+\left\|\mathbf{v}_{, 3}^{m}\right\|_{\left(L^{4}\left(\Omega^{m}\right)\right)^{3}}^{2} .
$$


Proposition 1. $I_{\mathrm{SV}}^{0}$ is continuous for the strong topology of $V$ and we have, for all $\mathbf{w} \in V:$

$$
\begin{aligned}
D I_{\mathrm{SV}}^{0}(\mathbf{u})(\mathbf{w})= & \int_{\Omega^{ \pm}}\left(\lambda^{ \pm} e_{k k}(\mathbf{u}) e_{k k}(\mathbf{w})+2 \mu^{ \pm} e_{i j}(\mathbf{u}) e_{i j}(\mathbf{w})\right) d \Omega \\
& +\frac{1}{2} \int_{\Omega^{m}}\left(\lambda^{m}+2 \mu^{m}\right)\left(\left(u_{1,3}^{2}+u_{2,3}^{2}\right)+\left(u_{3,3}+1\right)^{2}-1\right) \\
& \times\left(u_{1,3} w_{1,3}+u_{2,3} w_{2,3}+\left(u_{3,3}+1\right) w_{3,3}\right) d \Omega \\
& +\int_{\Omega^{m}} \mu^{m}\left(u_{1,3} w_{1,3}+u_{2,3} w_{2,3}\right) d \Omega-\int_{\Gamma_{f}} \mathbf{f}^{d} \cdot \mathbf{w} d \Gamma
\end{aligned}
$$

Remark 4. The functional $I_{\mathrm{SV}}^{0}$ is not convex; as a consequence, the continuity for the strong topology does not imply the lower semi-continuity for the weak topology.

(ii) Thanks to Proposition 1, the critical points $\mathbf{u} \in V$ of $I_{\mathrm{SV}}^{0}$ are solutions of the following variational problem: $\forall \mathbf{w} \in V$,

$$
(\mathcal{P})\left\{\begin{aligned}
\int_{\Omega^{ \pm}} & \left(\lambda^{ \pm} e_{k k}(\mathbf{u}) e_{k k}(\mathbf{w})+2 \mu^{ \pm} e_{i j}(\mathbf{u}) e_{i j}(\mathbf{w})\right) d \Omega \\
& +\frac{1}{2} \int_{\Omega^{m}}\left(\lambda^{m}+2 \mu^{m}\right)\left(\left(u_{1,3}^{2}+u_{2,3}^{2}\right)+\left(u_{3,3}+1\right)^{2}-1\right) \\
& \times\left(u_{1,3} w_{1,3}+u_{2,3} w_{2,3}+\left(u_{3,3}+1\right) w_{3,3}\right) d \Omega \\
& +\int_{\Omega^{m}} \mu^{m}\left(u_{1,3} w_{1,3}+u_{2,3} w_{2,3}\right) d \Omega=\int_{\Gamma_{f}} \mathbf{f}^{d} \cdot \mathbf{w} d \Gamma
\end{aligned}\right.
$$

The corresponding local equations are:

$$
\begin{gathered}
\begin{cases}\sigma_{i j}^{ \pm}=\lambda^{ \pm} e_{k k}\left(\mathbf{u}^{ \pm}\right) \delta_{i j}+2 \mu^{ \pm} e_{i j}\left(\mathbf{u}^{ \pm}\right) & \text {in } \Omega^{ \pm}, \\
\sigma_{i j, j}^{ \pm}=0 & \text { in } \Omega^{ \pm},\end{cases} \\
\begin{cases}\sigma_{\alpha 3}^{m}=\mu^{m} u_{\alpha, 3}^{m} & \text { in } \Omega^{m} \\
\sigma_{33}^{m}=\frac{1}{2}\left(\lambda^{m}+2 \mu^{m}\right)\left[\left(u_{1,3}^{m}\right)^{2}+\left(u_{2,3}^{m}\right)^{2}+\left(u_{3,3}^{m}+1\right)^{2}-1\right] & \text { in } \Omega^{m} \\
\left(\sigma_{i 3}^{m}+\sigma_{33}^{m} u_{i, 3}^{m}\right)_{, 3}=0 & \text { in } \Omega^{m}\end{cases} \\
\begin{cases}\mathbf{u}^{ \pm}=\mathbf{u}^{m} & \text { on } S^{ \pm}, \\
\sigma_{i 3}^{ \pm}=\sigma_{i 3}^{m}+\sigma_{33}^{m} u_{i, 3}^{m} & \text { on } S^{ \pm},\end{cases} \\
\begin{cases}\mathbf{u}^{ \pm}=0 & \text { on } \Gamma_{u}^{ \pm}, \\
\boldsymbol{\sigma}^{ \pm} \cdot \mathbf{n}=\mathbf{f}^{d} & \text { on } \Gamma_{f} .\end{cases}
\end{gathered}
$$

It appears that, at the first order, the behavior in the adherents is that of linear elasticity whereas it remains nonlinear in the adhesive. In order to prove the existence 
of at least one solution to problem $(\mathcal{P})$, we introduce the following functional

$$
\begin{aligned}
\hat{I}_{\mathrm{SV}}^{0}(\mathbf{v})= & \frac{1}{2} \int_{\Omega^{ \pm}}\left(\lambda^{+}\left(e_{k k}\left(\mathbf{v}^{ \pm}\right)\right)^{2}+2 \mu^{ \pm} e_{i j}\left(\mathbf{v}^{ \pm}\right) e_{i j}\left(\mathbf{v}^{ \pm}\right)\right) d \Omega \\
& +\frac{1}{2 h} \int_{S} \frac{\lambda^{m}+2 \mu^{m}}{4 h^{2}}\left(\left[v_{1}\right]^{2}+\left[v_{2}\right]^{2}+\left(\left[v_{3}\right]+h\right)^{2}-h^{2}\right)^{2} d \Omega \\
& +\frac{1}{2 h} \int_{S} \mu^{m}\left(\left[v_{1}\right]^{2}+\left[v_{2}\right]^{2}\right) d \Omega-\int_{\Gamma_{f}} \mathbf{f}^{d} \cdot \mathbf{v} d \Gamma,
\end{aligned}
$$

where $[\mathbf{v}]\left(x_{1}, x_{2}\right)=\mathbf{v}^{+}\left(x_{1}, x_{2}, \frac{h}{2}\right)-\mathbf{v}^{-}\left(x_{1}, x_{2},-\frac{h}{2}\right)$ defined on $S$, is the jump of the displacement field across $\Omega^{m}$. $\hat{I}_{\mathrm{SV}}^{0}$ is defined on the space

$$
\hat{V}=\left\{\mathbf{v}^{-} \in\left(H^{1}\left(\Omega^{-}\right)\right)^{3}, \mathbf{v}^{+} \in\left(H^{1}\left(\Omega^{+}\right)\right)^{3} ; \mathbf{v}^{ \pm}=0 \text { on } \Gamma_{u}^{ \pm}\right\},
$$

endowed with the norm $\|\mathbf{v}\|_{\hat{V}}$ such that $\|\mathbf{v}\|_{\hat{V}}^{2}=\left\|\mathbf{v}^{+}\right\|_{\left(H^{1}\left(\Omega^{+}\right)\right)^{3}}^{2}+\left\|\mathbf{v}^{-}\right\|_{\left(H^{1}\left(\Omega^{-}\right)\right)^{3}}^{2}$.

Proposition 2. If $\hat{\mathbf{u}} \in \hat{V}$ is a critical point of $\hat{I}_{\mathrm{SV}}^{0}$, then

$$
\mathbf{u}= \begin{cases}\hat{\mathbf{u}} & \text { in } \Omega^{ \pm}, \\ \frac{\hat{\mathbf{u}}^{+}}{h}\left(\frac{h}{2}+x_{3}\right)+\frac{\hat{\mathbf{u}}^{-}}{h}\left(\frac{h}{2}-x_{3}\right) & \text { in } \Omega^{m},\end{cases}
$$

is a regular solution of problem $(\mathcal{P})$.

Proof. Thanks to the continuous imbedding $\left(H^{1 / 2}(S)\right)^{3} \subset\left(L^{4}(S)\right)^{3}$, we verify that $\mathbf{u}$ defined by (4.13) belongs to $V$. Furthermore, if $\hat{\mathbf{u}}$ is a critical point of $\hat{I}_{\mathrm{SV}}^{0}$, then for all $\hat{\mathbf{w}} \in \hat{V}$,

$$
\begin{aligned}
\int_{\Omega^{ \pm}} & \left.\lambda^{ \pm} e_{k k}(\hat{\mathbf{u}}) e_{k k}(\hat{\mathbf{w}})+2 \mu^{ \pm} e_{i j}(\hat{\mathbf{u}}) e_{i j}(\hat{\mathbf{w}})\right) d \Omega \\
& +\frac{1}{h} \int_{S} \frac{\lambda^{m}+2 \mu^{m}}{2 h^{2}}\left(\left[\hat{u}_{1}\right]^{2}+\left[\hat{u}_{2}\right]^{2}+\left(\left[\hat{u}_{3}\right]+h\right)^{2}-h^{2}\right) \\
& \times\left(\left[\hat{u}_{1}\right]\left[\hat{w}_{1}\right]+\left[\hat{u}_{2}\right]\left[\hat{w}_{2}\right]+\left(\left[\hat{u}_{3}\right]+h\right)\left[\hat{w}_{3}\right]\right) d S \\
& +\frac{1}{h} \int_{S} \mu^{m}\left(\left[\hat{u}_{1}\right]\left[\hat{w}_{1}\right]+\left[\hat{u}_{2}\right]\left[\hat{w}_{2}\right]\right) d S=\int_{\Gamma_{f}} \mathbf{f}^{d} \cdot \mathbf{w} d \Gamma .
\end{aligned}
$$

Using (4.5) and (4.13), we have for $\mathbf{w} \in V$,

$$
\begin{aligned}
D I_{\mathrm{SV}}^{0}(\mathbf{u})(\mathbf{w})= & \int_{\Omega^{ \pm}}\left(\lambda^{ \pm} e_{k k}(\hat{\mathbf{u}}) e_{k k}(\hat{\mathbf{w}})+2 \mu^{ \pm} e_{i j}(\hat{\mathbf{u}}) e_{i j}(\hat{\mathbf{w}})\right) d \Omega \\
& +\frac{1}{2} \int_{\Omega^{m}}\left(\lambda^{m}+2 \mu^{m}\right)\left(\frac{1}{h^{2}}\left(\left[\hat{u}_{1}\right]^{2}+\left[\hat{u}_{2}\right]^{2}\right)+\left(\frac{\left[\hat{u}_{3}\right]}{h}+1\right)^{2}-1\right) \\
& \times\left(\frac{\left[\hat{u}_{1}\right]}{h} w_{1,3}+\frac{\left[\hat{u}_{2}\right]}{h} w_{2,3}+\left(\frac{\left[\hat{u}_{3}\right]}{h}+1\right) w_{3,3}\right) d \Omega \\
& +\int_{\Omega^{m}} \mu^{m}\left(\frac{\left[\hat{u}_{1}\right]}{h} w_{1,3}+\frac{\left[\hat{u}_{2}\right]}{h} w_{2,3}\right) d \Omega-\int_{\Gamma_{f}} \mathbf{f}^{d} \cdot \mathbf{w} d \Gamma
\end{aligned}
$$


Then, since $\mathbf{w} \in V$ implies $\hat{\mathbf{w}} \equiv \mathbf{w}_{\mid \Omega^{+} \cup \Omega^{-}} \in \hat{V}$, we deduce, identifying $S^{+}$and $S^{-}$ with $S$, and thanks to (4.14) and to the Fubini's theorem that $D I_{\mathrm{SV}}^{0}(\mathbf{u})(\mathbf{w})=0$ for all $\mathbf{w} \in V$.

(iii) Theorem 1. The functional $\hat{I}_{\mathrm{SV}}^{0}$ defined on the space $\hat{V}$ has at least one critical point.

Proof. To obtain the result, we show that $\hat{I}_{\mathrm{SV}}^{0}$ is lower bounded on $\hat{V}$ and reaches its infimum. Thanks to the Korn's inequality, there exist two reals $\alpha>0, \beta>0$ such that

$$
\hat{I}_{\mathrm{SV}}^{0}(\mathbf{v}) \geq \alpha\|\mathbf{v}\|_{\hat{V}}^{2}-\beta\left\|\mathbf{f}^{d}\right\|_{\left(L^{2}\left(\Gamma_{f}\right)\right)^{3}}\|\mathbf{v}\|_{\hat{V}}
$$

As a consequence, we have to show only the lower semi-continuity for the weak topology. From the relation:

$$
\begin{aligned}
& \left(\left[v_{1}\right]^{2}+\left[v_{2}\right]^{2}+\left[v_{3}+h\right]^{2}-h^{2}\right)^{2} \\
& \quad=\left(\left[v_{1}\right]^{2}+\left[v_{2}\right]^{2}+\left[v_{3}+h\right]^{2}\right)^{2}+h^{4}-2 h^{2}\left(\left[v_{1}\right]^{2}+\left[v_{2}\right]^{2}+\left[v_{3}+h\right]^{2}\right),
\end{aligned}
$$

$\hat{I}_{\mathrm{SV}}^{0}$ appears to be the sum of a convex and continuous terms for the strong topology, a continuous linear form and an additional term: $-\frac{1}{4 h} \int_{S}\left(\lambda^{m}+2 \mu^{m}\right)\left(\left[v_{1}\right]^{2}+\left[v_{2}\right]^{2}+\right.$ $\left.\left[v_{3}+h\right]^{2}\right) d S$. The compactness of the injection $H^{1 / 2}(S) \subset L^{2}(S)$ implies that this additional term is weakly continuous. Since a convex and continuous function is also weakly lower semi-continuous, $\hat{I}_{\mathrm{SV}}^{0}$ is lower semi-continuous. The minimum is then reached.

(iv) Identifying $S^{+}$and $S^{-}$with $S$, the transmission conditions on $S$ become, for any critical point of $\hat{I}_{\mathrm{SV}}^{0}$ :

$$
\left\{\begin{array}{l}
\sigma_{\alpha 3}^{+}=\sigma_{\alpha 3}^{-}=\frac{\mu^{m}}{h}\left[u_{\alpha}\right]+\frac{\lambda^{m}+2 \mu^{m}}{2 h^{3}}\left(\left[u_{1}\right]^{2}+\left[u_{2}\right]^{2}+\left(\left[u_{3}\right]+h\right)^{2}-h^{2}\right)\left[u_{\alpha}\right], \\
\sigma_{33}^{+}=\sigma_{33}^{-}=\frac{\lambda^{m}+2 \mu^{m}}{2 h^{3}}\left(\left[u_{1}\right]^{2}+\left[u_{2}\right]^{2}+\left(\left[u_{3}\right]+h\right)^{2}-h^{2}\right)\left(\left[u_{3}\right]+h\right) .
\end{array}\right.
$$

From (4.13) and (4.18) it follows that any critical point of $\hat{I}_{\mathrm{SV}}^{0}$ gives rise to a solution of the problem with linear displacements and constant stresses in the adhesive.

The structure of the system (4.18) suggests that, for some choice of $\mathbf{f}^{d}$, the functional $\hat{I}_{\mathrm{SV}}^{0}$ could have many critical points. For $\mathbf{f}^{d} \equiv 0, \hat{\mathbf{v}}=0$ is the unique minimum. Furthermore, we have:

$$
\begin{aligned}
D^{2} \hat{I}_{\mathrm{SV}}^{0}(\mathbf{v})(\mathbf{w}, \hat{\mathbf{w}})= & \int_{\Omega^{ \pm}}\left(\lambda^{ \pm} e_{k k}(\mathbf{w}) e_{k k}(\hat{\mathbf{w}})+2 \mu^{ \pm} e_{i j}(\mathbf{w}) e_{i j}(\hat{\mathbf{w}})\right) d \Omega \\
& +\frac{1}{h} \int_{S} \mu^{m}\left(\left[w_{1}\right]\left[\hat{w}_{1}\right]+\left[w_{2}\right]\left[\hat{w}_{2}\right]\right) d S \\
& +\frac{1}{2 h^{3}} \int_{S}\left(\lambda^{m}+2 \mu^{m}\right)\left(\left[v_{1}\right]^{2}+\left[v_{2}\right]^{2}+\left(\left[v_{3}\right]+h\right)^{2}-h^{2}\right)
\end{aligned}
$$




$$
\begin{aligned}
& \times\left(\left[w_{1}\right]\left[\hat{w}_{1}\right]+\left[w_{2}\right]\left[\hat{w}_{2}\right]+\left[w_{3}\right]\left[\hat{w}_{3}\right]\right) d S \\
& +\frac{1}{h^{3}} \int_{S}\left(\lambda^{m}+2 \mu^{m}\right)\left(\left[v_{1}\right]\left[\hat{w}_{1}\right]+\left[v_{2}\right]\left[\hat{w}_{2}\right]+\left(\left[v_{3}\right]+h\right)\left[\hat{w}_{3}\right]\right) \\
& \times\left(\left[v_{1}\right]\left[w_{1}\right]+\left[v_{2}\right]\left[w_{2}\right]+\left(\left[v_{3}\right]+h\right)\left[w_{3}\right]\right) d S
\end{aligned}
$$

leading to

$$
\begin{aligned}
D^{2} \hat{I}_{\mathrm{SV}}^{0}(\mathbf{0})(\mathbf{w}, \hat{\mathbf{w}})= & \int_{\Omega^{ \pm}}\left(\lambda^{ \pm} e_{k k}(\mathbf{w}) e_{k k}(\hat{\mathbf{w}})+2 \mu^{ \pm} e_{i j}(\mathbf{w}) e_{i j}(\hat{\mathbf{w}})\right) d \Omega \\
& +\frac{1}{h} \int_{S} \mu^{m}\left(\left[w_{1}\right]\left[\hat{w}_{1}\right]+\left[w_{2}\right]\left[\hat{w}_{2}\right]\right) d S \\
& +\frac{1}{h} \int_{S}\left(\lambda^{m}+2 \mu^{m}\right)\left[w_{3}\right]\left[\hat{w}_{3}\right] d S
\end{aligned}
$$

which is a definite positive isomorphism. This implies that $\hat{I}_{\mathrm{SV}}^{0}$ is strictly convex at $\mathbf{0}$ and so, for $\left\|\mathbf{f}^{d}\right\|_{\left(L^{2}\left(\Gamma_{f}\right)\right)^{3}}$ small enough, the functional $\hat{I}_{\mathrm{SV}}^{0}$ has a unique absolute minimum. Similar arguments can be used on the functional $I_{\mathrm{SV}}^{0}$ to prove

Proposition 3. There exists $\delta>0$ such that for $\left\|\mathbf{f}^{d}\right\|_{\left(L^{2}\left(\Gamma_{f}\right)\right)^{3}}<\delta$, the problem $(\mathcal{P})$ has a unique solution, which is the absolute minimum of $I_{\mathrm{SV}}^{0}$ on $V$.

(v) In order to study the non-uniqueness of the solution of $(\mathcal{P})$, let us consider the application $G: \mathbb{R}^{3} \rightarrow \mathbb{R}^{3} ;\left(x_{1}, x_{2}, x_{3}\right) \rightarrow\left(G_{1}, G_{2}, G_{3}\right)$ defined by:

$$
\left\{\begin{array}{l}
G_{1}\left(x_{1}, x_{2}, x_{3}\right)=\mu^{m} x_{1}+\frac{\lambda^{m}+2 \mu^{m}}{2} x_{1}\left(x_{1}^{2}+x_{2}^{2}+\left(x_{3}+1\right)^{2}-1\right), \\
G_{2}\left(x_{1}, x_{2}, x_{3}\right)=\mu^{m} x_{2}+\frac{\lambda^{m}+2 \mu^{m}}{2} x_{2}\left(x_{1}^{2}+x_{2}^{2}+\left(x_{3}+1\right)^{2}-1\right), \\
G_{3}\left(x_{1}, x_{2}, x_{3}\right)=\frac{\lambda^{m}+2 \mu^{m}}{2}\left(x_{1}^{2}+x_{2}^{2}+\left(x_{3}+1\right)^{2}-1\right)\left(1+x_{3}\right) .
\end{array}\right.
$$

Then, Eqs. (4.8) and (4.9) become simply:

$$
\begin{cases}\mathbf{u}^{ \pm}=\mathbf{u}^{m} & \text { on } S^{ \pm}, \\ G\left(u_{, 3}^{m}\right)=\boldsymbol{\sigma}^{+} \cdot \mathbf{e}_{3}=\boldsymbol{\sigma}^{-} \cdot \mathbf{e}_{3} & \text { in } \Omega^{m} .\end{cases}
$$

If, $\mathbf{f}^{d}$ being fixed, this system determine $u_{, 3}^{m}$ in a unique way, then $\mathbf{u}^{m}$ is linear with respect to $x_{3}$ in $\Omega^{m}$ and it is so completely determined by [u]. This solution corresponds to the regular one obtained from Proposition 2. If, on the contrary, the system (4.22) possesses several solutions, then, we can take $\mathbf{u}^{m}$ piecewise linear with respect to the variable $x_{3}$ on arbitrary subintervals of $]-\frac{h}{2}, \frac{h}{2}$ [. These solutions do not correspond to critical points of $\hat{I}_{\mathrm{SV}}^{0}$. An example of this situation is given by the case $\boldsymbol{\sigma}^{+} \cdot \mathbf{e}_{3}=0$. In this case, the system (4.22) has the solutions 


$$
\begin{gathered}
u_{i, 3}^{m}=0, \\
u_{\alpha, 3}^{m}=0, \quad u_{3,3}^{m}=-1, \\
\left(u_{1,3}^{m}\right)^{2}+\left(u_{2,3}\right)^{2}=\frac{\lambda^{m}}{\lambda^{m}+2 \mu^{m}}, \quad u_{3,3}^{m}=-1 .
\end{gathered}
$$

If $\mathbf{f}^{d} \equiv \mathbf{0}$, the unique solution is $u_{i, 3}^{m}=0$, which corresponds to the regular solution of Proposition 2, and so $I_{\mathrm{SV}}^{0}$ has a strict minimum. Besides, thanks to a straightforward computation of the second derivative, we can easily show that the other solutions do not correspond to a strict minimum of $I_{\mathrm{SV}}^{0}$. We conjecture that this situation is general: the minima of $I_{\mathrm{SV}}^{0}$ are (regular) critical points associated to the minima of $\hat{I}_{\mathrm{SV}}^{0}$.

\section{Study of the Limit Problem: The Ciarlet-Geymonat Case}

(i) The functional $I_{\mathrm{CG}}^{0}(3.10)$ is defined on the set:

$$
\begin{aligned}
U= & \left\{\mathbf{v}=\left(\mathbf{v}^{-}, \mathbf{v}^{m}, \mathbf{v}^{+}\right) ; \mathbf{v} \in\left(L^{2}(\Omega)\right)^{3}, \mathbf{v}^{-} \in\left(H^{1}\left(\Omega^{-}\right)\right)^{3}, \mathbf{v}^{+} \in\left(H^{1}\left(\Omega^{+}\right)\right)^{3}\right. \\
& v_{k, 3}^{m} \in L^{2}\left(\Omega^{m}\right),\left(1+v_{3,3}^{m}\right)>0 \text { p.p. in } \Omega^{m}, \log \left(1+v_{3,3}^{m}\right) \in L^{1}\left(\Omega^{m}\right) \\
& \left.\mathbf{v}_{\mid S+}^{+}=\mathbf{v}_{\mid S^{+}}^{m}, \mathbf{v}_{\mid S-}^{-}=\mathbf{v}_{\mid S^{-}}^{m}, \mathbf{v}=0 \text { on } \Gamma_{u}^{ \pm}\right\}
\end{aligned}
$$

By virtue of the convexity of the function $x \rightarrow x-\log (1+x), U$ is a non-empty $(\mathbf{0} \in U)$ convex set of the Hilbert's space:

$$
\begin{aligned}
W= & \left\{\mathbf{v}=\left(\mathbf{v}^{-}, \mathbf{v}^{m}, \mathbf{v}^{+}\right) ; \mathbf{v} \in\left(L^{2}(\Omega)\right)^{3}, \mathbf{v}^{-} \in\left(H^{1}\left(\Omega^{-}\right)\right)^{3}, \mathbf{v}^{+} \in\left(H^{1}\left(\Omega^{+}\right)\right)^{3},\right. \\
& \left.v_{k, 3}^{m} \in L^{2}\left(\Omega^{m}\right), \mathbf{v}_{\mid S^{+}}^{+}=\mathbf{v}_{\mid S^{+}}^{m}, \mathbf{v}_{\mid S^{-}}^{-}=\mathbf{v}_{\mid S^{-}}^{m}, \mathbf{v}=0 \text { on } \Gamma_{u}^{ \pm}\right\}
\end{aligned}
$$

endowed with the natural norm $\|\mathbf{v}\|_{W}$ such that

$$
\|\mathbf{v}\|_{W}^{2}=\left\|\mathbf{v}^{+}\right\|_{\left(H^{1}\left(\Omega^{+}\right)\right)^{3}}^{2}+\left\|\mathbf{v}^{-}\right\|_{\left(H^{1}\left(\Omega^{-}\right)\right)^{3}}^{2}+\left\|\mathbf{v}^{m}\right\|_{\left(L^{2}\left(\Omega^{m}\right)\right)^{3}}^{2}+\left\|\mathbf{v}_{, 3}^{m}\right\|_{\left(L^{2}\left(\Omega^{m}\right)\right)^{3}}^{2} .
$$

Following the approach of J. Ball (see Ref. 7), we extend the domain of definition of $I_{\mathrm{CG}}^{0}$ to $W$. To this end, let us define the function $g: \mathbb{R} \rightarrow \overline{\mathbb{R}} \equiv \mathbb{R} \cup\{ \pm \infty\}$ :

$$
g(\zeta)= \begin{cases}\left(\lambda^{m}+2 \mu^{m}\right)\left(\frac{1}{2} \zeta^{2}+\zeta-\log (1+\zeta)\right) & \text { if } 1+\zeta>0 \\ +\infty & \text { if } 1+\zeta \leq 0\end{cases}
$$

Remark 5. $g$ is a Carathéodory's function.

Lemma 1. The functional

$$
\mathbf{v} \rightarrow F(\mathbf{v})=\int_{\Omega^{m}} g(\mathbf{v}(\mathbf{x})) d \mathbf{x},
$$

is convex, positive and weakly lower semi-continuous from $L^{2}\left(\Omega^{m}\right)$ to $\overline{\mathbb{R}}$. 
Proof. Since $g(\zeta) \geq 0$ and is a Carathéodory's function, the functional $F$ is positive and lower semi-continuous from $L^{2}\left(\Omega^{m}\right)$ to $\overline{\mathbb{R}}$ (see Ref. 10). Furthermore, $\zeta \rightarrow g(\zeta)$ is convex and so $\mathbf{v} \rightarrow F(\mathbf{v})$ is convex.

(ii) Theorem 2. There exists at least an element $\mathbf{u} \in U$ such that:

$$
I_{\mathrm{CG}}^{0}(\mathbf{u})=\operatorname{Inf}_{v \in U} I_{\mathrm{CG}}^{0}(\mathbf{v}) .
$$

Proof. From $\mathbf{0} \in U$ and $I_{\mathrm{CG}}^{0}(\mathbf{0})=0$, we deduce that:

$$
\operatorname{Inf}_{v \in U} I_{\mathrm{CG}}^{0}(\mathbf{v}) \leq 0 \text {. }
$$

We then extend the definition of the functional $I_{\mathrm{CG}}^{0}$ to the space $W$ by defining:

$$
\begin{aligned}
\bar{I}_{\mathrm{CG}}^{0}(\mathbf{v})= & \int_{\Omega^{ \pm}}\left(\frac{\lambda^{ \pm}}{2}\left(e_{k k}\left(\mathbf{v}^{ \pm}\right)\right)^{2}+\mu^{ \pm} e_{i j}\left(\mathbf{v}^{ \pm}\right) e_{i j}\left(\mathbf{v}^{ \pm}\right)\right) d \Omega+\frac{1}{2} F\left(v_{3,3}^{m}\right) \\
& +\frac{1}{2} \int_{\Omega^{m}} \mu^{m}\left(\left(v_{1,3}^{m}\right)^{2}+\left(v_{2,3}^{m}\right)^{2}\right) d \Omega-\int_{\Gamma_{f}} \mathbf{f}^{d} \cdot \mathbf{v} d \Gamma .
\end{aligned}
$$

We remark that $\bar{I}_{\mathrm{CG}}^{0}(\mathbf{v})<\infty \Rightarrow \mathbf{v} \in U$. Indeed, if $\bar{I}_{\mathrm{CG}}^{0}(\mathbf{v})<\infty$, then $F\left(v_{3,3}^{m}\right)=$ $\int_{\Omega^{m}} g\left(v_{3,3}^{m}(\mathbf{x})\right) d \mathbf{x}<\infty$ and so $1+v_{3,3}^{m}>0$ p.p. in $\Omega^{m}$. Besides,

$$
\log \left(1+v_{3,3}^{m}\right)=\frac{1}{2}\left(v_{3,3}^{m}\right)^{2}+v_{3,3}^{m}-\frac{1}{\lambda^{m}+2 \mu^{m}} g\left(v_{3,3}^{m}(x)\right) \in L^{1}\left(\Omega^{m}\right),
$$

thanks to $v_{3,3}^{m} \in L^{2}\left(\Omega^{m}\right), g\left(v_{3,3}^{m}\right) \in L^{1}\left(\Omega^{m}\right)$ and $\frac{1}{\lambda^{m}+2 \mu^{m}} \in L^{\infty}\left(\Omega^{m}\right)$. We deduce then

$$
\bar{I}_{\mathrm{CG}}^{0}(\mathbf{v})=I_{\mathrm{CG}}^{0}(\mathbf{v})<\infty \Longleftrightarrow \mathbf{v} \in U .
$$

Then, thanks to Lemma $1, \bar{I}_{\mathrm{CG}}^{0}$ is convex, weakly lower semi-continuous on $W$ in $\overline{\mathbb{R}}$. Since $g(\zeta) \geq \frac{1}{2}\left(\lambda^{m}+2 \mu^{m}\right) \zeta^{2}$, for $\zeta>-1$, we deduce, like in Ref. 2, that there exist two reals $\alpha>0, \beta>0$ such that:

$$
\bar{I}_{\mathrm{CG}}^{0}(\mathbf{v}) \geq \alpha\|\mathbf{v}\|_{W}^{2}-\beta\left\|\mathbf{f}^{d}\right\|_{L^{2}\left(\Gamma_{f}\right)}\left\|\mathbf{v}^{ \pm}\right\|_{\left(H^{1}\left(\Omega^{ \pm}\right)\right)^{3}} .
$$

Hence, there exists $\mathbf{u} \in W$ such that $\bar{I}_{\mathrm{CG}}^{0}(\mathbf{u})=\operatorname{Inf}_{v \in W} \bar{I}_{\mathrm{CG}}^{0}(\mathbf{v}) \leq 0$. From (5.10), $\mathbf{u} \in U$ showing that $\bar{I}_{\mathrm{CG}}^{0}$ reaches (and so $I_{\mathrm{CG}}^{0}$ ) its minimum on $\mathbf{u} \in U$ :

$$
I_{\mathrm{CG}}^{0}(\mathbf{u})=\inf _{v \in U} I_{\mathrm{CG}}^{0}(\mathbf{v})=\inf _{v \in W} \bar{I}_{\mathrm{CG}}^{0}(\mathbf{v}) .
$$

Proposition 4. The minimum $\mathbf{u}$ of $I_{\mathrm{CG}}^{0}$ is unique.

Proof. The result is a direct consequence of the strict convexity of the application $\zeta \rightarrow g(\zeta)$ for $1+\zeta>0$.

When the solution $\mathbf{u}$ satisfies the regularity condition $\left(1+u_{3,3}^{m}\right)^{-1} \in L^{\infty}\left(\Omega^{m}\right)$, then $\bar{I}_{\mathrm{CG}}^{0}$ admits a directional derivative for all $\mathbf{v} \in W_{\infty}=\left\{\mathbf{v} \in W ; v_{3,3}^{m} \in\right.$ $\left.L^{\infty}\left(\Omega^{m}\right)\right\}$. Moreover the minimum $\mathbf{u}$ satisfies the following variational equation for all $\mathbf{v} \in W_{\infty}$ : 


$$
(\mathcal{Q})\left\{\begin{aligned}
\int_{\Omega^{ \pm}} & \left(\lambda^{ \pm} e_{k k}\left(\mathbf{u}^{ \pm}\right) e_{k k}\left(\mathbf{v}^{ \pm}\right)+2 \mu^{ \pm} e_{i j}\left(\mathbf{u}^{ \pm}\right) e_{i j}\left(\mathbf{v}^{ \pm}\right)\right) d \Omega \\
& +\frac{1}{2} \int_{\Omega^{m}}\left(\lambda^{m}+2 \mu^{m}\right)\left(1+u_{3,3}^{m}-\frac{1}{1+u_{3,3}^{m}}\right) v_{3,3}^{m} d \Omega \\
& +\int_{\Omega^{m}} \mu^{m}\left(u_{1,3}^{m} v_{1,3}^{m}+u_{2,3}^{m} v_{2,3}^{m}\right) d \Omega-\int_{\Gamma_{f}} \mathbf{f}^{d} \cdot \mathbf{v} d \Gamma=0 .
\end{aligned}\right.
$$

(iii) As in the Saint Venant-Kirchhoff case, it is possible to define the functional

$$
\begin{aligned}
\hat{I}_{\mathrm{CG}}^{0}(\mathbf{v})= & \frac{1}{2} \int_{\Omega^{ \pm}}\left(\lambda^{ \pm}\left(e_{k k}\left(\mathbf{v}^{ \pm}\right)\right)^{2}+2 \mu^{ \pm} e_{i j}\left(\mathbf{v}^{ \pm}\right) e_{i j}\left(\mathbf{v}^{ \pm}\right)\right) d \Omega \\
& +\frac{1}{2 h} \int_{S}\left(\lambda^{m}+2 \mu^{m}\right)\left(\frac{1}{2}\left[v_{3}\right]^{2}+h\left[v_{3}\right]-h^{2} \log \left(1+\frac{\left[v_{3}\right]}{h}\right)\right) d S \\
& +\frac{1}{2 h} \int_{S} \mu^{m}\left(\left[v_{1}\right]^{2}+\left[v_{2}\right]^{2}\right) d S-\int_{\Gamma_{f}} \mathbf{f}^{d} \cdot \mathbf{v} d \Gamma
\end{aligned}
$$

on the set

$$
\begin{aligned}
\hat{U}= & \left\{\mathbf{v}^{-} \in\left(H^{1}\left(\Omega^{-}\right)\right)^{3}, \mathbf{v}^{+} \in\left(H^{1}\left(\Omega^{+}\right)\right)^{3}, 1+\frac{\left[v_{3}\right]}{h}>0 \text { p.p. on } S,\right. \\
& \left.\log \left(1+\frac{\left[v_{3}\right]}{h}\right) \in L^{1}(S), \mathbf{v}^{ \pm}=0 \text { on } \Gamma_{u}^{ \pm}\right\}
\end{aligned}
$$

which is a nonempty convex subset of the Hilbert space:

$$
\hat{W}=\left\{\mathbf{v}^{-} \in\left(H^{1}\left(\Omega^{-}\right)\right)^{3}, \mathbf{v}^{+} \in\left(H^{1}\left(\Omega^{+}\right)\right)^{3} ; \mathbf{v}^{ \pm}=0 \text { on } \Gamma_{u}^{ \pm}\right\},
$$

endowed with the natural norm $\|\mathbf{v}\|_{\hat{W}}^{2}=\left\|\mathbf{v}^{+}\right\|_{\left(H^{1}\left(\Omega^{+}\right)\right)^{3}}^{2}+\left\|\mathbf{v}^{-}\right\|_{\left(H^{1}\left(\Omega^{-}\right)\right)^{3}}^{2}$. Then, in a similar way to Lemma 1, we have:

Lemma 2. Let $\hat{g}$ be the Carathéodory's function defined as:

$$
\hat{g}(\zeta)= \begin{cases}\left(\lambda^{m}+2 \mu^{m}\right)\left(\frac{1}{2} \zeta^{2}+h \zeta-h^{2} \log \left(1+\frac{\zeta}{h}\right)\right) & \text { if } 1+\frac{\zeta}{h}>0, \\ +\infty & \text { if } 1+\frac{\zeta}{h} \leq 0 .\end{cases}
$$

Then, the functional $\mathbf{v} \rightarrow \hat{F}(\mathbf{v})=\int_{S} \hat{g}(\mathbf{v}(\mathbf{x})) d S$ is convex positive and weakly lower semi-continuous from $L^{2}(S)$ to $\overline{\mathbb{R}}$.

As in the proof of Theorem 2, we extend the definition of $\hat{I}_{\mathrm{CG}}^{0}(\mathbf{v})$ to the space $\hat{W}$ with:

$$
\begin{aligned}
\tilde{I}_{\mathrm{CG}}^{0}(\mathbf{v})= & \frac{1}{2} \int_{\Omega^{ \pm}}\left(\lambda^{ \pm}\left(e_{k k}\left(\mathbf{v}^{ \pm}\right)\right)^{2}+2 \mu^{ \pm} e_{i j}\left(\mathbf{v}^{ \pm}\right) e_{i j}\left(\mathbf{v}^{ \pm}\right)\right) d \Omega \\
& +\frac{1}{2 h} \int_{S} \mu^{m}\left(\left[v_{1}\right]^{2}+\left[v_{2}\right]^{2}\right) d S+\frac{1}{2 h} \hat{F}\left(\left[v_{3}\right]\right)-\int_{\Gamma_{f}} \mathbf{f}^{d} \cdot \mathbf{v} d \Gamma .
\end{aligned}
$$

Then, in a similar approach to Theorem 2 and Proposition 4, we obtain 
Theorem 3. There exists a unique element $\hat{\mathbf{u}} \in \hat{U}$ such that:

$$
\hat{I}_{\mathrm{CG}}^{0}(\hat{\mathbf{u}})=\inf _{v \in \hat{U}} \hat{I}_{\mathrm{CG}}^{0}(\mathbf{u})=\inf _{v \in \hat{W}} \tilde{I}_{\mathrm{CG}}^{0}(\mathbf{v}) .
$$

When the solution $\hat{\mathbf{u}}$ satisfies the regularity condition $\left(h+\left[\hat{u}_{3}\right]\right)^{-1} \in L^{4 / 3}(S)$, then $\hat{I}_{\mathrm{CG}}^{0}$ is Gâteaux-differentiable at $\hat{\mathbf{u}}$. Moreover, the minimum $\hat{\mathbf{u}}$ can be characterized by the following variational equation $\forall \mathbf{v} \in \hat{W}$ :

$$
(\hat{\mathcal{Q}})\left\{\begin{aligned}
\int_{\Omega^{ \pm}} & \left(\lambda^{ \pm} e_{k k}\left(\hat{\mathbf{u}}^{ \pm}\right) e_{k k}\left(\mathbf{v}^{ \pm}\right)+2 \mu^{ \pm} e_{i j}\left(\hat{\mathbf{u}}^{ \pm}\right) e_{i j}\left(\mathbf{v}^{ \pm}\right)\right) d \Omega \\
& +\frac{1}{2 h} \int_{S}\left(\lambda^{m}+2 \mu^{m}\right)\left(h+\left[\hat{u}_{3}\right]-\frac{1}{h+\left[\hat{u}_{3}\right]}\right)\left[v_{3}\right] d S \\
& +\frac{1}{h} \int_{S} \mu^{m}\left(\left[\hat{u}_{1}\right]\left[v_{1}\right]+\left[\hat{u}_{2}\right]\left[v_{2}\right]\right) d S-\int_{\Gamma_{f}} \mathbf{f}^{d} \mathbf{v} d \Gamma=0 .
\end{aligned}\right.
$$

(iv) Proposition 5. If $\hat{\mathbf{u}} \in \hat{U}$ verifies (5.17) then

$$
\tilde{\mathbf{u}}= \begin{cases}\hat{\mathbf{u}} & \text { in } \Omega^{ \pm}, \\ \frac{\hat{\mathbf{u}}^{+}}{h}\left(\frac{h}{2}+x_{3}\right)+\frac{\hat{\mathbf{u}}^{-}}{h}\left(\frac{h}{2}-x_{3}\right) & \text { in } \Omega^{m},\end{cases}
$$

is the minimum of $I_{\mathrm{CG}}^{0}$.

Proof. (i) Let us verify that $\tilde{\mathbf{u}} \in U$. Indeed, if $\tilde{\mathbf{u}} \in \hat{U}$, then $\tilde{\mathbf{u}}$ defined by (5.18) verifies $\tilde{\mathbf{u}}^{m}=\frac{\hat{u}^{+}}{h}\left(\frac{h}{2}+x_{3}\right)+\frac{\hat{u}^{-}}{h}\left(\frac{h}{2}-x_{3}\right) \in L^{2}\left(\Omega^{m}\right), \tilde{\mathbf{u}}_{, 3}^{m}=\frac{[\hat{u}]}{h} \in\left(L^{2}\left(\Omega^{m}\right)\right)^{3}, 1+\tilde{u}_{3,3}^{m}=$ $1+\frac{\left[\hat{u}_{3}\right]}{h}>0$ p.p. in $\Omega^{m}$ and $\int_{\Omega^{m}} \log \left(1+\tilde{u}_{3,3}^{m}\right) d \Omega^{m}=h \int_{S} \log \left(1+\frac{\left[\hat{u}_{3}\right]}{h}\right) d S<\infty$.

(ii) Finally, let us show that

$$
\tilde{I}_{\mathrm{CG}}^{0}(\tilde{\mathbf{u}}) \leq \tilde{I}_{\mathrm{CG}}^{0}(\mathbf{v}) \quad \forall \mathbf{v} \in U .
$$

We associate to $\mathbf{v}$ the function $\tilde{\mathbf{v}}$ as follows:

$$
\tilde{\mathbf{v}}= \begin{cases}\tilde{\mathbf{v}}^{ \pm}=\mathbf{v}^{ \pm} & \text {in } \Omega^{ \pm}, \\ \tilde{\mathbf{v}}^{m}=\frac{\mathbf{v}^{+}}{h}\left(\frac{h}{2}+x_{3}\right)+\frac{\mathbf{v}^{-}}{h}\left(\frac{h}{2}-x_{3}\right) & \text { in } \Omega^{m} .\end{cases}
$$

Then, using the equality $\tilde{\mathbf{v}}_{3}^{m}=\frac{[v]}{h}=\frac{1}{h} \int_{-\frac{h}{2}}^{\frac{h}{2}} \mathbf{v}_{, 3} d x_{3}$ and the convexity of $\zeta \rightarrow g(\zeta)$, we write

$$
g\left(\tilde{v}_{3,3}^{m}\right) \leq \frac{1}{h} \int_{-\frac{h}{2}}^{\frac{h}{2}} g\left(v_{3,3}^{m}\right) d x_{3} .
$$

For $\mathbf{v} \in W$, we note $\hat{\mathbf{v}} \in \hat{W}$ the restriction of $\mathbf{v}$ to $\Omega^{+}$and $\Omega^{-}$. An explicit computation leads, with $(5.20)$ and $(5.21)$, to $\tilde{I}_{\mathrm{CG}}^{0}(\hat{\mathbf{v}}) \leq \tilde{I}_{\mathrm{CG}}^{0}(\tilde{\mathbf{v}}) \leq \tilde{I}_{\mathrm{CG}}^{0}(\mathbf{v})$ and so, thanks to (5.17), we get for any $\mathbf{v} \in W$ :

$$
\hat{I}_{\mathrm{CG}}^{0}(\hat{\mathbf{u}})=I_{\mathrm{CG}}^{0}(\tilde{\mathbf{u}}) \leq \tilde{I}_{\mathrm{CG}}^{0}(\hat{\mathbf{v}}) \leq \tilde{I}_{\mathrm{CG}}^{0}(\mathbf{v}) .
$$




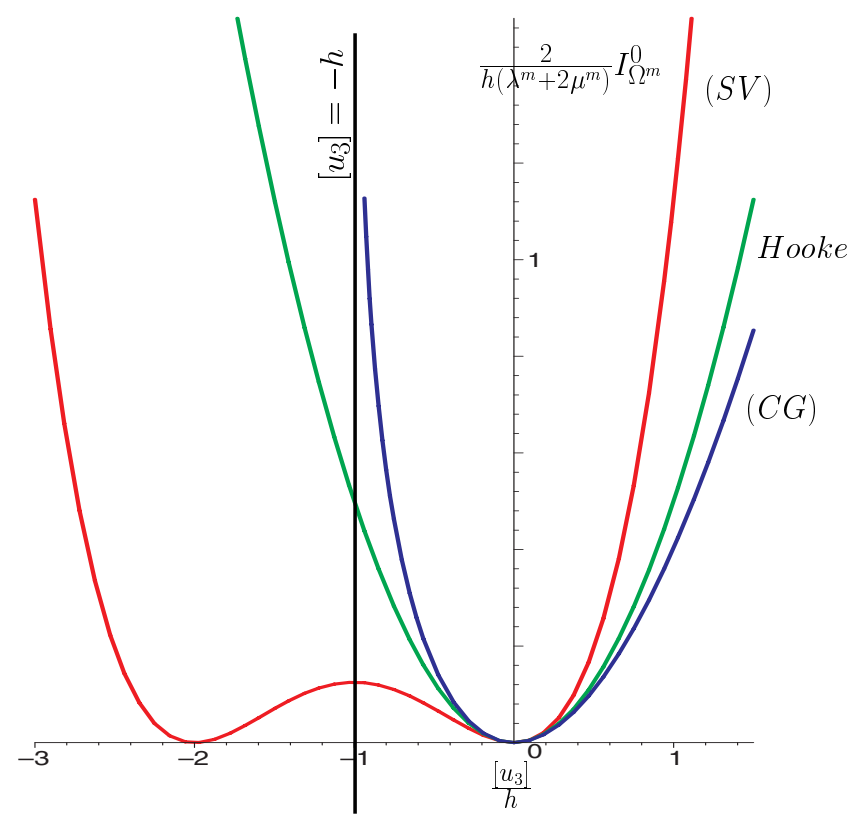

Fig. 2. Strain energies $I_{\Omega^{m}}^{0}$ associated to $\breve{W}_{\mathrm{SV}}(\mathbf{E})[(S V)]$, to $\breve{W}_{\mathrm{CG}}(\mathbf{E})[(C G)]$ and to $\breve{W}_{\mathrm{SV}}(\mathbf{e})$ [Hooke].

\section{Comparison in the One-Dimensional Case}

In the one-dimensional case, we consider a structure composed of three bars. The adherents have a length $h$ and the adhesive bar has a length $\varepsilon h$. We consider only the most significant case when the solution is linear in the adhesive. In the Saint Venant-Kirchhoff case, thanks to Proposition 2, the energy of the solution in the adhesive is:

$$
I_{\Omega^{m}}^{0}\left(u_{3}\right)=\frac{h}{2}\left(\lambda^{m}+2 \mu^{m}\right)\left(\frac{\left[u_{3}\right]}{h}+\frac{1}{2}\left(\frac{\left[u_{3}\right]}{h}\right)^{2}\right)^{2} .
$$

The transmission conditions (4.9) become simply

$$
\sigma_{33}^{ \pm}=\left(\lambda^{m}+2 \mu^{m}\right)\left(\frac{\left[u_{3}\right]}{h}+\frac{1}{2}\left(\frac{\left[u_{3}\right]}{h}\right)^{2}\right)\left(1+\frac{\left[u_{3}\right]}{h}\right) .
$$

In the Ciarlet-Geymonat case, we obtain in a similar way, by virtue of Proposition 5

$$
I_{\Omega^{m}}^{0}\left(u_{3}\right)=\frac{h}{2}\left(\lambda^{m}+2 \mu^{m}\right)\left(\frac{\left[u_{3}\right]}{h}+\frac{1}{2}\left(\frac{\left[u_{3}\right]}{h}\right)^{2}-\log \left(1+\frac{\left[v_{3}\right]}{h}\right)\right) .
$$

The transmission conditions can be written as:

$$
\sigma_{33}^{ \pm}=\left(\frac{\lambda^{m}}{2}+\mu^{m}\right)\left(1+\frac{\left[u_{3}\right]}{h}-\frac{1}{1+\frac{\left[u_{3}\right]}{h}}\right) .
$$




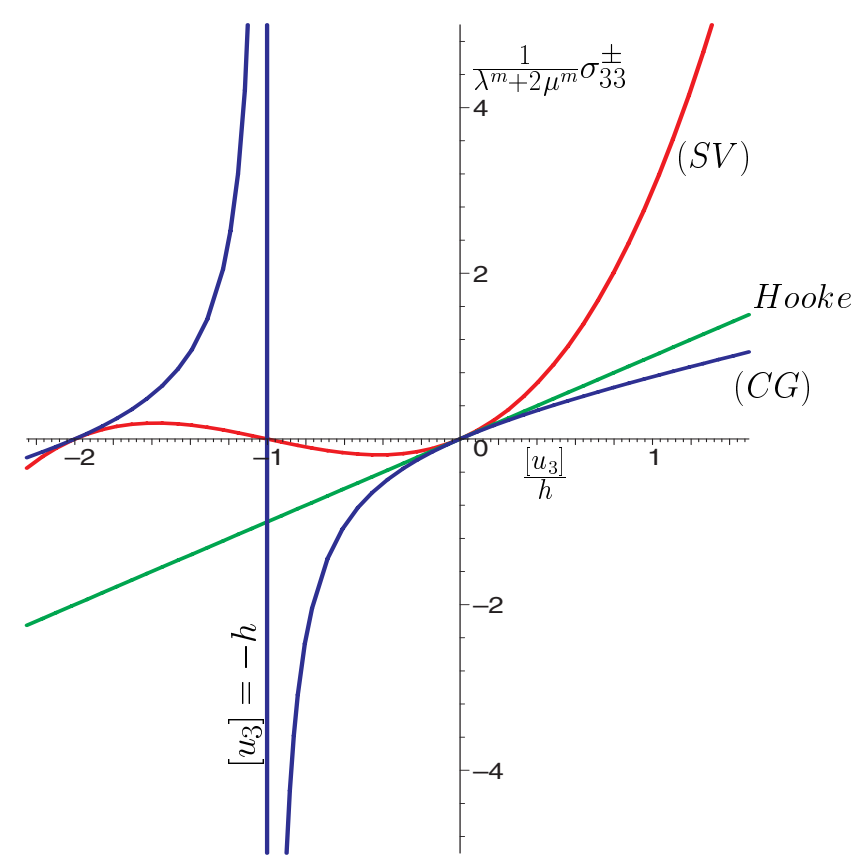

Fig. 3. Stress $\sigma_{33}^{ \pm}$associated to $\breve{W}_{\mathrm{SV}}(\mathbf{E})[(S V)]$, to $\breve{W}_{\mathrm{CG}}(\mathbf{E})[(C G)]$ and to $\breve{W}_{\mathrm{SV}}(\mathbf{e})[$ Hooke].

Figure 2 represents the limit strain energy associated to the adhesive, up to the constant $\frac{2}{h\left(\lambda^{m}+2 \mu^{m}\right)}$ as a function of the dimensionless quantity $\frac{\left[u_{3}\right]}{h}$, for the two stored energy functions. The figure depicts also the limit energy obtained with the stored energy function $\breve{W}_{\mathrm{SV}}(\mathbf{e})=\frac{\lambda}{2}(\operatorname{Tr} \mathbf{e})^{2}+\mu \operatorname{Tr}\left(\mathbf{e}^{2}\right): I_{\Omega^{m}}^{0}\left(u_{3}\right)=\frac{h}{2}\left(\lambda^{m}+\right.$ $\left.2 \mu^{m}\right)\left(\frac{\left[u_{3}\right]}{h}\right)^{2}$. As expected with the relation (2.7), the three energies coincide for small strains $\frac{\left[u_{3}\right]}{h}$. Furthermore, the graphs clearly show that the strain energy associated to $\breve{W}_{\mathrm{SV}}$ is not convex (Remark 4$)$ and that the strain energy associated to $\breve{W}_{\mathrm{CG}}$ has a vertical asymptote: the flattening of the adhesive $\left(\left[u_{3}\right]=-h\right)$ would require an infinite strain energy.

Similar conclusions can be obtained from the evolution of $\sigma_{33}^{ \pm}$(up to the constant $\left.\frac{1}{\lambda^{m}+2 \mu^{m}}\right)$ as a function of the strain $\frac{\left[u_{3}\right]}{h}$ described in Fig. 3. The stress associated to $\breve{W}_{\mathrm{CG}}$ is an increasing function of $\frac{\left[u_{3}\right]}{h}$ for $\left[u_{3}\right]>-h$. At last, the behavior of the material of Ciarlet-Geymonat appears softer, for a traction load $\left(\frac{\left[u_{3}\right]}{h}>0\right)$ than Saint Venant-Kirchhoff materials, and conversely stiffer for a compressive load $\left(\frac{\left[u_{3}\right]}{h}<0\right)$.

\section{A Numerical Example: The Single-Lap Joint}

In this paragraph, the limit models are tested on the single-lap joint structure, often used as a typical example (see e.g. Ref. 11). As shown in Fig. 4, the structure consists of two aluminum plates and of a thin adhesive layer of epoxy type. The material 


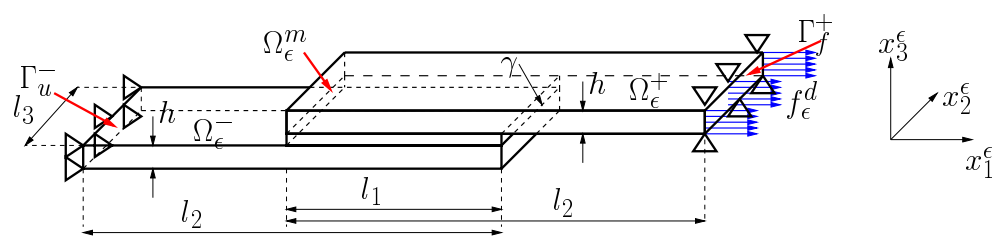

Fig. 4. The single-lap joint: $h=3 \mathrm{~mm}, l_{1}=60 \mathrm{~mm}, l_{2}=120 \mathrm{~mm}, l_{3}=30 \mathrm{~mm}$, adhesive thickness $\varepsilon h=0.3 \mathrm{~mm}$.

Table 1. Material characteristics.

\begin{tabular}{lcc}
\hline & $E(\mathrm{MPa})$ & $\nu$ \\
\hline Adherents & 70,000 & 0.3 \\
Adhesive & 3,000 & 0.36 \\
\hline
\end{tabular}

characteristics are reported in Table 1 . The structure is fixed on $\Gamma_{u}^{-} \subset \partial \Omega_{\varepsilon}^{-}$. On $\Gamma_{f}^{+}$ the component $u_{3}$ is equal to zero, the normal load component $f_{1}$ is given equal to $8 \mathrm{MPa}$ and the tangential load component in the direction $\mathbf{e}_{2}$ is equal to zero.

A finite element method is used to determine critical points of $\hat{I}_{\mathrm{SV}}^{0}$ and $\hat{I}_{\mathrm{CG}}^{0}$ in the space $\hat{V}$ and $\hat{U}$ respectively (a numerical resolution of the problem associated to $I_{\mathrm{CG}}^{\varepsilon}$ is presented in Ref. 12). More precisely, a finite element with 16 degrees of freedom is used: the shape functions are quadratic with respect to the in-plane variables $\left(x_{1}, x_{2}\right)$ and linear with respect to the out-of-plane variable $x_{3}$. When the limit models are used, the joint $\Omega_{\varepsilon}^{m}$ is substituted by its mid-surface $S$, where all the nodes are merged in the reference configuration. At last, the numerical algorithm is based on a Newton's method and is displacement controlled.

For the single-lap joint structure, a mesh of 3,246 nodes is considered. Figures 5 and 6 depict the jumps $\left[u_{1}\right]$ and $\left[u_{3}\right]$ respectively, along the line $\gamma=\left\{\left(x_{1}=60, x_{2} \in\right.\right.$ $\left.\left.\left[0, l_{3}\right], x_{3}=0\right)\right\} \subset \partial S \cap \partial \Omega^{-} \cap \partial \Omega^{+}$(see Fig. 4), obtained for the limit models (noted (SV) for the Saint Venant-Kirchhoff case $\left(\hat{I}_{\mathrm{SV}}^{0}\right)$ and noted $(\mathrm{CG})$ for the CiarletGeymonat case $\left.\left(\hat{I}_{\mathrm{CG}}^{0}\right)\right)$. The two limit problems give close results: the limit model associated to $\breve{W}_{\mathrm{CG}}^{\varepsilon}$ is slightly softer than the model associated to $\breve{W}_{\mathrm{SV}}^{\varepsilon}$. The figures depict also the jumps, obtained from the initial problem associated to $\breve{W}_{\mathrm{SV}}^{\varepsilon}$. In this case, the joint was meshed using one element in the thickness. The difference, which remains small, is more important for the first component (about an error of 8\%) than for the third one (less than 1\%). This result can be explained by the fact that the nonlinear terms of the plane strains $E_{\alpha \beta}$ and the transverse shear strains $E_{\alpha 3}$, predominant here, are neglected in the limit models. The predominant strain is $E_{13}$ and reaches approximately the value 0.035 on the line $\gamma$. The differences for the second components $\left[u_{2}\right]$ are very small and are not presented. The results obtained with more than one element in the thickness of the adhesive are very closed.

At last, the aim of Fig. 7 is to compare more precisely the results of the limit model with the results of the initial model. Only the Saint Venant-Kirchhoff case is 
20 F. Krasucki, A. Münch \& Y. Ousset

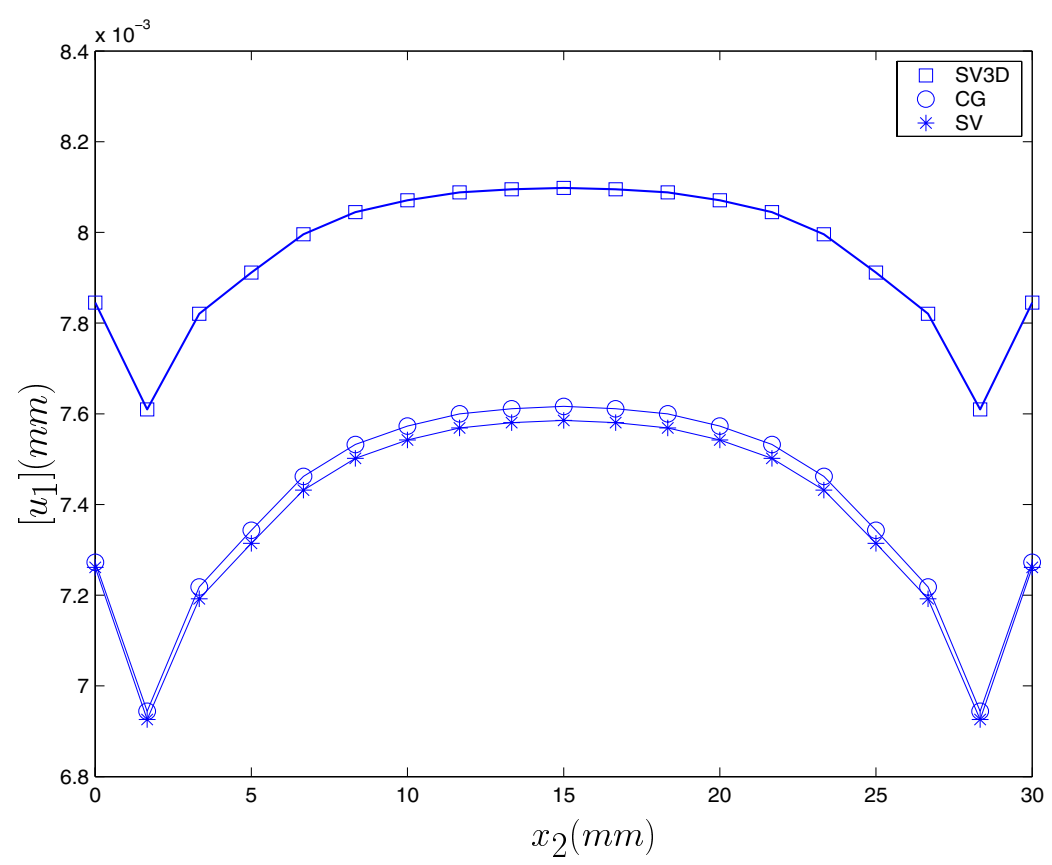

Fig. 5. Jump $\left[u_{1}\right]$ along the line $\gamma=\left(60, x_{2}, 0\right)$.

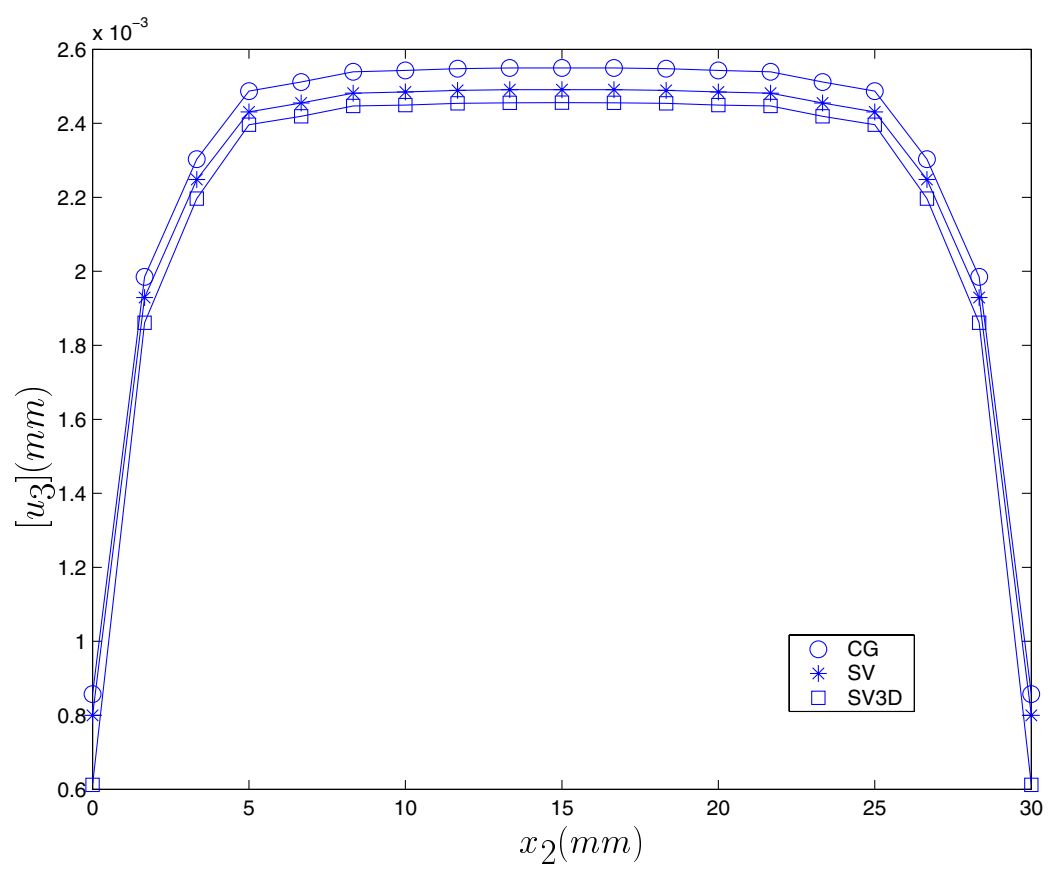

Fig. 6. Jump $\left[u_{3}\right]$ along the line $\gamma=\left(60, x_{2}, 0\right)$. 


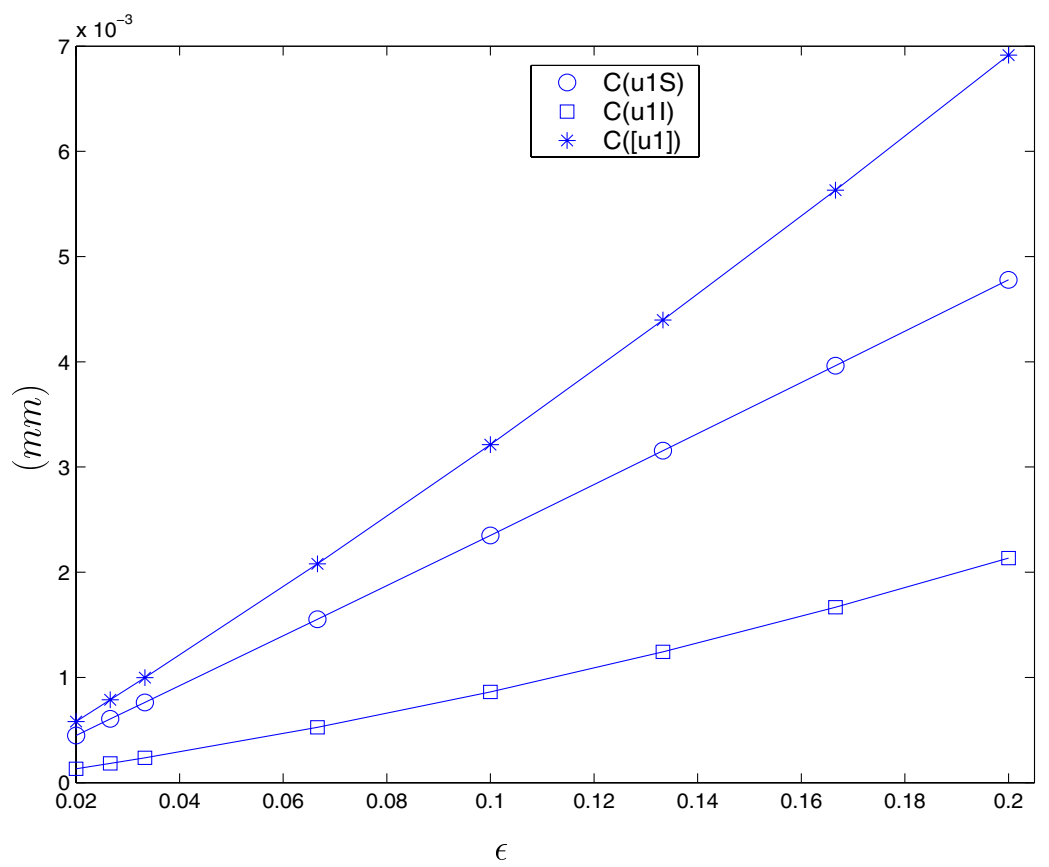

Fig. 7. Comparisons between the limit and complete model (Saint Venant-Kirchhoff case).

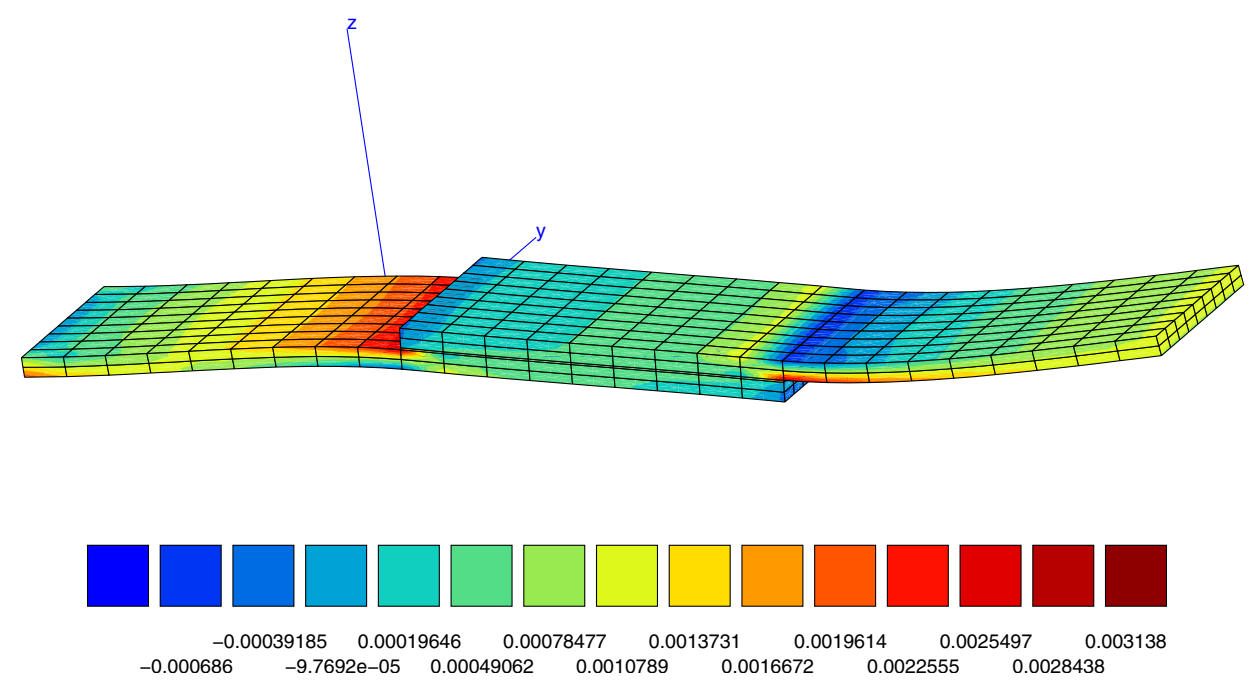

Fig. 8. Configuration of the single lap joint at equilibrium (isovalues represent the strain $E_{11}$ ). 
discussed. The figure depicts, as a function of the parameter $\varepsilon$, the gap on the jump $\left[u_{1}\right](60,15,0)$ between the limit and the initial model. This gap is noted $C[u 1]$ on Fig. 7. The figure depicts also the quantities $C(u 1 S)$ and $C(u 1 I)\left(u 1 S\right.$ designs $u_{1}^{+}$, $u 1 I$ designs $u_{1}^{-}$and let us recall that $\left.\left[u_{1}\right]=u_{1}^{+}-u_{1}^{-}\right)$. As expected, the three gaps converge toward zero when the parameter $\varepsilon$ tends to zero. The rates of convergence can be evaluated. We obtain:

$$
C([u 1])=O\left(\varepsilon^{1.072}\right), \quad C(u 1 S)=O\left(\varepsilon^{1.026}\right), \quad C(u 1 I)=O\left(\varepsilon^{1.202}\right) .
$$

These rates of convergence are in complete agreement with the formal expansion (3.5) and suggest that the limit model is an approximation of order $\varepsilon$ of the initial model. Finally, Fig. 8 represents the configuration of the structure at equilibrium.

\section{References}

1. M. Goland and E. Reissner, The stresses in cemented joints, J. Appl. Mech. ASME 11 (1944) A17-A27.

2. G. Geymonat, F. Krasucki and S. Lenci, Mathematical analysis of a bonded joint with a soft thin adhesive, Math. Mech. Solids 4 (1999) 201-255.

3. C. Licht and G. Michaille, Une modélisation du comportement d'un joint collé élastique, C. R. Acad. Sci. Paris Série I 322 (1996) 295-300.

4. P. G. Ciarlet, Mathematical Elasticity, Vol. I: Three Dimensional Elasticity (NorthHolland, 1988).

5. P. G. Ciarlet and G. Geymonat, Sur les lois de comportement en élasticité non linéaire compressible, C. R. Acad. Sci. Paris Série II 295 (1982) 423-426.

6. F. Krasucki, A. Münch and Y. Ousset, Asymptotic analysis of a bonded joint in nonlinear elasticity, C. R. Acad. Sci. Paris Série IIb 329 (2001) 429-434.

7. J. Ball, Convexity conditions and existence theorems in nonlinear elasticity, Arch Rational Mech. Anal. 63 (1977) 337-403.

8. F. Krasucki and A. Münch, Modèles de joint collé en élasticité non linéaire, 15ième Congrès Français de Mécanique (Nancy, 2001).

9. P. G. Ciarlet and P. Destuynder, A justification of a nonlinear model in plate theory, Comput. Meth. Appl. Mech. Engrg. 17/18 (1979) 227-258.

10. I. Ekeland and R. Teman, Analyse Convexe et Problèmes Variationnels (DunodGauthier Villars, 1974).

11. U. Edlund and A. Klarbring, A geometrically nonlinear model of the adhesive joint problem and its numerical treatment, Comput. Meth. Appl. Mech. Engrg. 96 (1992) 329-350.

12. P. Le Tallec and M. Vidrascu, Une méthode numérique pour les problèmes d'équilibre de corps hyperélastiques compressibles en grandes déformations, Numer. Math. 43 (1984) 199-224. 